\title{
El desarrollo de las competencias sobre política científica en Estados europeos: evaluación y retos
}

Laura Díez Bueso

Miriam Cueto Pérez

Ana Fernández-Zubieta

\begin{abstract}
Sumario: I. Introducción.-II. Alemania. II.1. Responsabilidades estatales en investigación e innovación. II.2. La gobernanza de la ciencia. II.3. La planificación de la política científica. II.4. Colaboración científica público-privada y transferencia de conocimiento y resultados.III. Austria. III.1. Responsabilidades estatales en investigación e innovación. III.2. La gobernanza de la ciencia. III.3. La planificación de la política científica. III.4. Colaboración científica público-privada y de la transferencia de conocimiento y resultados. -IV. Reino Unido. IV.1. Responsabilidades estatales en investigación e innovación. IV.2. La gobernanza de la ciencia. IV.3. La planificación de la política científica. IV.4. Colaboración científica público-privada y transferencia de conocimiento y resultado. -V. España. V.1. Responsabilidades estatales en investigación e innovación. V.2. La gobernanza de la ciencia. V.3. La planificación de la política científica. V.4. Colaboración científica público-privada y transferencia de conocimiento y resultados.VI. Conclusiones
\end{abstract}

\section{Introducción}

La política científica se ha convertido en materia prioritaria para la Unión Europea (UE), marcadamente en las dos últimas décadas. Desde el Consejo Europeo de Lisboa de 2000, cuyo objetivo estratégico fue convertir la economía de la Unión en "la economía del conocimiento más competitiva del mundo", han sido constantes las medidas encaminadas a reforzar la investigación, el desarrollo y la innovación (I+D+i) a nivel europeo y también a potenciar los sistemas científicos de los Estados miembros. En esta dirección, con la entrada en vigor del Tratado de Lisboa en 2009 se refuerzan las capacidades de las instituciones europeas en política científica. 
La actual coyuntura de crisis económica y financiera, no ha sido considerada por parte de la Unión ni de la Organización para la Cooperación y el Desarrollo Económico (OCDE) como un momento para retroceder en la inversión en ciencia, sino como una oportunidad para fomentar la política científica centrándola en los grandes retos sociales, en orden a la creación de puestos de trabajo y para favorecer el crecimiento económico (1). Esta es la idea que fundamenta la Innovation Union aprobada en 2010 y la European Research Area (ERA) de 2012, enmarcadas ambas en la estrategia Europa 2020 para el crecimiento inteligente, sostenible e integrador (2).

Avanzando en esta línea, la UE ha promovido las Research Innovation Strategies for Smart Specialisation-RIS3 con la intención de que los Estados prioricen su actividad económica en base a sus ventajas competitivas y su masa crítica(3). Concretamente, para el periodo 2014-2020, la Comisión Europea ha definido un enfoque integrado de todos los fondos de la política de cohesión, con una fijación clara de prioridades y de resultados a alcanzar; en esta dirección, la Comisión obliga a los países y a las regiones a elaborar sus RIS3 centrándose en las especializaciones económicas y de conocimiento que se ajusten mejor a su potencial de innovación, en base a los activos y las capacidades del territorio. La Comisión ha fijado, incluso, la metodología que se debe emplear para aprobar las RIS3, con la voluntad de asegurar que durante el proceso de elaboración de las mismas puedan identificarse debidamente los ámbitos de especialización (4).

(1) También los países con sistemas científicos punteros han considerado que la crisis actual es un momento adecuado para potenciar la ciencia focalizándola en los retos de la sociedad, como se explica en MALKIN, D., El sistema català d'innovació. Reptes i orientació de les polítiques publiques. Consell General de Chambres de Catalunya, 2009, p. 5.

(2) En 2010 la Comisión Europea (CE) publica la Europa 2020, que prioriza cinco objetivos en materia de empleo, innovación, educación, integración social y clima-energía. Para coordinar los esfuerzos en investigación e innovación, la CE desarrolló la Innovation Union y creó la ERA, con la intención de implicar tanto a la Unión como a sus miembros en la mejora del sistema público de la ciencia, centrando la investigación en los grandes retos sociales para promover el crecimiento y la creación de puestos de trabajo. Sobre esta última y destacable iniciativa puede verse QUINTANA, O.: «El Espacio Europeo de Investigación: una Europa donde los investigadores y el conocimiento puedan circular sin fronteras». Informe CYD 2012. La contribución de las universidades españolas al desarrollo. Fundación Conocimiento y Desarrollo, 2013, pp. 209-211.

(3) Sobre este concepto de smart specialisation aplicado I+D+i verse Baier, E., KROLL, H. y ZENKER, A., Templates of smart specialisation: experience of placed-based regional development strategies in Germany and Austria. Fraunhofer, 2013; este documento incluye también un análisis de la especialización inteligente en el caso de las regiones alemanas y austríacas.

(4) La Comisión Europea aprobó la Guide to research and innovation strategies for smart specialisation-RIS3 establecido una metodología bidireccional e interactiva (combina los enfoques de arriba abajo - top-down - y de abajo arriba - bottom-up-); parte de las evidencias; y se constituye en base a consensos entre los agentes del sistema. 
El escenario descrito aboca a la necesidad de realizar un estudio sobre los sistemas científicos de los países europeos para situar el caso español en este contexto (5). Concretamente, conviene analizar el desarrollo estatal de la política científica en España y otros países de referencia en esta materia, como son Alemania, Austria y Reino Unido. La selección de estos países se explica, no sólo por su carácter políticamente descentralizado, sino también por su tradición y eficiencia en el campo de la política científica. En este sentido, el Innovation Union Scoreboard (IUS) de 2014, que clasifica a los Estados de la UE en cuatro grupos según su actuación en materia de innovación, sitúa a Alemania en el primer grupo como Innovation Leader y a Austria y a Reino Unido en el segundo como Innovation Followers, en tanto que sitúa a España en el tercero como Moderate Innovator. El estudio pretende, pues, que las referencias del Derecho comparado sean países europeos con una estructura territorial compuesta y con una destacable actuación en el campo de la política científica.

En cuanto a la selección de los temas a evaluar es conocido el gran abanico de cuestiones que, de forma directa o tangencial, configuran un sistema científico. No obstante, si se revisan los abundantes informes relativos a la I+D+i en Europa, todos coinciden en identificar como retos los asuntos relacionados con la gobernanza de la ciencia, con la planificación científica y con la colaboración público-privada y la transferencia de conocimientos y resultados (6).

\section{Alemania}

\section{II.1. Responsabilidades estatales en investigación e innovación}

La distribución de competencias en materia de política científica entre la Federación y los dieciséis Länder se establece en el artículo 74.13 de la Constitución, donde se determina que «la legislación concurrente se extiende al fomento de la investigación científica». En el artículo 72.1 se concreta que «en el ámbito de la legislación concurrente, los Länder tienen la facultad de legislar en tanto que la Federación no haya hecho uso me-

(5) Un análisis exhaustivo sobre la política científica en diversos países y regiones europeos puede encontrarse en DÍEZ BUESO, L., Los sistemas de investigación en los países europeos descentralizados. Estudio comparado de España, Alemania, Austria y Bélgica y de Cataluña, Baviera, Estiria y las Regiones belgas. Institut d'Estudis Autonòmics, 2013.

(6) Entre mucha otra bibliografía, sobre estos retos puede leerse European Research Area. Progress Report 2014. Directorate General for Research and Innovation, European Union, 2014. 
diante ley de su competencia legislativa» (7). De este modo, en Alemania el protagonismo en materia de ciencia se comparte inicialmente entre el Estado y los Länder, aunque las competencias relativas al mercado laboral y a la propiedad industrial e intelectual se concentran principalmente en la Federación y las competencias fiscales regionales son limitadas. Un dato esencial para entender este reparto de competencias es el elevado grado de actuaciones conjuntas entre los dos niveles de poder, que básicamente se traducen en programas o iniciativas comunes donde la política científica regional complementa las iniciativas nacionales o las que tienen proyección internacional. Esta cooperación abarca, no sólo la aprobación de programas, sino también su financiación, que se lleva a cabo conjuntamente. Uno de los ejemplos más elocuentes es la Initiative for Excellence, acordada en 2005 entre el gobierno federal y el de los Länder y financiada hasta el 2017, que dota de recursos con base competitiva a escuelas de graduados, clusters y universidades de cara a mejorar su competitividad internacional (8). La cofinanciación de los Institutos no Universitarios de Investigación (los conocidos como PROSA que equivaldrían a nuestros organismos públicos de investigación, OPIS) es otro elemento vertebrador del sistema alemán de la ciencia, específicamente la cofinanciación de los dedicados a la ciencia aplicada, como los Fraunhofer-Institutes, ya que se trata de institutos muy relevantes para la orientación del desarrollo científico regional. De hecho, los gobiernos regionales presionan para atraer estos institutos en su región con medidas favorables de financiación (9).

Como se puede intuir, la intensa cooperación ha provocado que tanto Federación como los Länder centren sus prioridades de política científica en ámbitos diferentes. Por ejemplo, en el campo de la innovación los Länder, no sólo han incrementado su gasto, sino que se han concentrado en las obligaciones asumidas en los programas conjuntos con la Federación; como consecuencia, las políticas regionales en innovación han ganado importancia como instrumento de diferenciación y de competición entre las mismas regiones. Por su parte, en el ámbito de la innovación del Estado ha centrado, o bien en programas de infraestructuras transversales, o bien en programas especializados en financiar tecnologías en un nivel

(7) Una descripción del sistema de distribución competencial alemán puede encontrarse en ARROYO, A., «Fundamentos constitucionales del reparto de competencias en la República Federal Alemana». Cooperación y reparto competencial en los Estados descentralizados, Instituto de Estudios Autonómicos, 2013.

(8) Una valoración muy positiva sobre la eficacia este programa puede encontrarse en el informe del Expert Commission on Research and Innovation-EFI 2014, p. 21.

(9) Así se explica en STAHLECK, TY BAIER, E., Analysis of the regional dimensiones of investment in research. Case study regional report: Bavaria (Alemania). ERAWATCH, 2007, p. 17. 
pre-competitivo(10). Se ha llegado a afirmar que, gracias a este sistema de cooperación y debido a las diferencias en el desarrollo de la política científica de los Länder, los solapamientos ocurren ocasionalmente; sin embargo, si se analiza concretamente la coordinación existente entre la Federación y algunos de los Länder con más actividad en política científica, se ha sostenido igualmente la necesidad de mejorar esta coordinación ya que se detectan ciertas redundancias(11). En cuanto a la coordinación orgánica, hay que señalar que no existía ningún consejo que coordinara las políticas científicas de Federación y Länder hasta 2008, cuando se crea la Joint Science Conference (GWK)(12); entre otras tareas, este órgano elabora informes que evalúan los logros y los retos del Pact for Research and Innovation, otro ejemplo de colaboración al que posteriormente se aludirá.

\section{II.2. La gobernanza de la ciencia}

El sistema de gobernanza en Alemania está muy desarrollado y es estable (13). Empezando por el nivel estatal y respecto de los órganos de dirección, el Ministerio de Educación e Investigación (BMBF) es el responsable principal de la política científica: de este Ministerio depende la mayor parte de la planificación y de la financiación de la ciencia en el país, tanto en lo referente a programas estatales como programas coordinados con los Länder o la financiación destinado a órganos, instituciones o empresas dedicados a la I+D+i. Concretamente, promueve la investigación básica y apoya a las instituciones que la llevan a cabo; fomenta la investigación en los sectores del medio ambiente, el clima, la ecología, la salud y la educación; y apoya tecnologías clave en campos muy diversos, como la movilidad o el empleo. Otro importante actor a nivel de dirección

(10) Sobre esta dualidad en la política científica alemana puede verse WILSON, D. y SOUITARIS, V., "Do Germany s Federal and Land Governments (still) co-ordinate their innovation policías?». Research Policías, núm. 31, pp. 1123 hasta 1140.

(11) Los mismos autores que defienden la escasez de solapamientos relativizan esta afirmación, por ejemplo, para Baviera en STAHLECK,T.Y BAIER, E., Analysis of the regional ..., cit., Pp. 11 y 38.

(12) En este artículo se ha optado por citar el nombre de órganos, instituciones y programas en inglés y añadir las siglas correspondientes al alemán por dos motivos. El primero, para una más amplia comprensión del texto, dado que la mayor parte de sus lectores estarán familiarizados con el inglés y no con el alemán. El segundo, porque si se desea hacer una búsqueda en internet para ampliar información sobre los órganos, instituciones y programas citados ésta puede realizarse utilizando la nomenclatura inglesa, pero no la española (puesto que todos las páginas en alemán tienen su correspondiente versión inglesa pero no española).

(13) Un cuadro explicativo de todos los agentes implicados en la gobernanza científica alemana puede encontrarse en Voigt, P., ERAWATCH country reports 2013: Germany. European Commission, 2014, p. 8. 
de la política científica es el Ministerio de Economía y Tecnología (BMWi), que también planifica y financia programas en I+D+i. Estos programas se centran particularmente en el apoyo a las Pequeñas y Medianas Empresas (PYMEs) y el emprendimiento, promoviendo medidas centradas en la innovación, la cooperación en la investigación y el consulting tecnológico. Además, aprueba y financia programas de investigación energética, aeroespacial y aeronáutica; financia algunos PROSA; y financia también, junto con los Länder, programas focalizados en la innovación. La coordinación entre los dos ministerios protagonistas en política científica, BMBF y BMWi, ha sido tradicionalmente limitada; aunque hay procedimientos formales bajo la responsabilidad superior del BMBF, éstos se circunscriben básicamente al intercambio de información. Sin embargo y como se apuntará, la coordinación mejoró de forma sustancial con la puesta en marcha en 2010 de la High-Tech Strategy 2020 for Germany (HTS 2020), con un enfoque interdepartamental que involucra a los diferentes ministerios y sus políticas.

A nivel asesor hay tres órganos: la Expert Commission on Research and Innovation (EFI), que asesora a la Cancillería Federal; el German Science Council (WR), que asesora tanto al gobierno estatal como a los Länder, además de realizar regularmente evaluaciones; y la IndustryScience Research Alliance, que presenta de manera habitual propuestas y recomendaciones relacionadas con las líneas de actuación recogidas en la HTS 2020. En cuanto a la gestión de la política científica, en Alemania hay varias agencias que se encuentran mayoritariamente situadas en los grandes centros de investigación y que administran la mayor parte de los programas financiados por fondos públicos. Estas agencias se gestionan conjuntamente por Estado y Länder a través del Programme Administrating Agencias (Projektträger). En el terreno de la gestión de la financiación, hay también el KfW Banking Group, banca pública federal $(80 \%)$ y regional $(20 \%)$ que otorga créditos y garantías a las empresas privadas.

Respecto a la implementación de la política científica, en Alemania existen unas 400 instituciones de educación superior que se distribuyen de la siguiente manera. Unas 100 universidades aglutinan las dos terceras partes de los estudiantes universitarios ofreciendo una educación teórica y científica y la posibilidad de realizar posteriormente un doctorado; paralelamente, hay unas 200 universidades de ciencias aplicadas con el otro tercio de matriculados, que al encontrarse orientadas a la educación aplicada no ofrecen todos los campos o disciplinas de estudio. El resto son universidades especializadas (como, por ejemplo, las Politécnicas o escuelas de arte). Junto a las anteriores están en funcionamiento unas 120 universidades privadas cuyo número se ha incrementado en los últimos años de forma importante y cuya oferta académica se centra espe- 
cialmente en las ciencias aplicadas(14). Aún en el nivel de la implementación de la ciencia, en Alemania hay un gran abanico de PROSA. Estos tienen una significación en el sistema de investigación cuantitativamente comparable al de las universidades. Se trata de un conjunto de organizaciones con una gran variedad de modelos de gobernanza y con un papel diverso en el sistema científico, que va desde institutos de investigación básica pura, centrados en la educación doctoral a institutos de investigación aplicada dirigidos a la cooperación con la industria. Su papel esencial consiste en proveer de una investigación orientada a largo plazo realizada en grandes infraestructuras y en ofrecer a los científicos destacados un espacio para concentrarse en la investigación reduciendo su carga docente. De acuerdo con su financiación, estos PROSA se clasifican en tres grupos: de financiación federal, de los Länder o conjunto; desde una perspectiva cualitativa, son cuatro las grandes organizaciones en el sector de los pros, todos ellos de financiación mixta: la Max Planck Society (MPG), la Fraunhofer Society (FHG) y la Leibniz Association (WGL). A pesar de esta clasificación que distingue entre universidades y PROSA, estos dos actores del sistema se encuentran estrechamente ligados. Para empezar, la mayoría de los directores de los PROSA son a la vez profesores permanentes en las universidades y ejercen cargos en las mismas; igualmente, muchos investigadores, los PROS, imparten clases en la universidad. Además, los principales PROS se encuentran activamente implicados en la educación de grado y de postgrado, especialmente en la docencia a doctorandos.

\section{II.3. La planificación de la política científica}

La política científica en Alemania ha sido estable y predecible gracias a los programas plurianuales y a un amplio portafolio de instrumentos de financiación. Sin embargo, no fue hasta la aprobación de la High-Tech Strategy para el período 2006-2009 cuando, por primera vez, se establecieron objetivos, prioridades y medidas concretas que implicaban a todos los ministerios del gobierno. Este plan fue actualizado en 2010 a través de la HTS 2020, que concentra los recursos públicos en I+D+i y mejora la coordinación interministerial. Esta nueva Estrategia sostiene que el desarrollo científico y tecnológico y la innovación son el medio para afrontar los retos globales que la misma identifica: el cambio climático y de-

(14) Sobre la gobernanza de las universidades alemanas puede leerse, DE BOER, H., ENDERS, J. y SCHIMANK, U., "¿Hacia una nueva gestión pública? La gobernanza de los sistemas universitarios en Inglaterra, Los Países Bajos, Austria y Alemania». KEHM, B. (comp.), La nueva gobernanza de los sistemas universitarios. Octaedro, ICE-UB, 2012, pp. 193-214. 
mográfico; la propagación de enfermedades comunes; la escasez de alimentos a nivel mundial; y la limitación de los recursos materiales y energéticos.

Resulta muy interesante apuntar que, desde 2007, BMBF Foresight ofrece cada dos años previsiones prospectivas en materia de ciencia y señala las necesidades sociales de futuro en términos de $\mathrm{I}+\mathrm{D}+\mathrm{i}$, con un horizonte a quince años vista; así, abarca una amplia mirada hacia adelante de cara a ofrecer guías para la agenda de prioridades científicas. Haciendo un gran paso más en esta dirección, recientemente se ha creado un interesante instrumento de planificación de la política científica, los Proyectos orientados de futuro (Zukunsftsprojekte): dado que una de las principales finalidades de la HTS 2020 es guía la I+D+i hacia un número concreto de metas, el gobierno federal aprueba estos Proyectos como herramienta básica para alcanzarlas, ya que diseñan una hoja de ruta que conduce hacia la consecución de metas parciales. En marzo de 2012, el Ejecutivo aprobó el HTS 2020 Action Plan, con diez Proyectos orientados de futuro que detallan objetivos específicos que deben alcanzarse en un plazo de 10 a 15 años. La relevancia de estos instrumentos prospectivos ha sido señalada en el informe del Experto Commission on Research and Innovation-EFI 2011, en el sentido de que el éxito del HTS 2020 dependerá básicamente de su proceso de implementación (15).

En Alemania la planificación también se lleva a cabo conjuntamente entre Federación y Länder a través de programas plurianuales, entre ellos, el Higher Education Pact 2020, aprobado en 2007 y reeditado en 2009 hasta 2015, aunque diseñado para continuar hasta 2020; este Pacto tiene como objetivo asegurar la educación superior a la vista del crecimiento del volumen de estudiantes e incluye financiación adicional para infraestructuras universitarias de investigación, además recoge el compromiso de los Länder de promover partenariados entre las instituciones universitarias y las empresas. Otro ejemplo de planificación conjunta es el Pact for Research and Innovation, firmado en 2005 con el fin de alcanzar el $3 \%$ del gasto en $I+D+i$ y que se amplió al $5 \%$ para los años 2011 a 2015; con este Pacto se pretende asegurar la financiación y hacer un mejor uso de los recursos existentes, concentrando la excelencia, incrementando la cooperación y las redes, apoyando a los jóvenes científicos y haciendo posible aproximaciones científicas no convencionales.

Aparte de esta programación conjunta, la Federación puso en marcha dos instrumentos más con una repercusión directa y decisiva en la planificación de la política científica de los Länder: los programas EXIST y Bio-

(15) Expert Commission on Research and Innovation-EFI 2011, p. 13. 
regio Contest(16). El primero se inició en 1998 y se centra en mejorar el clima para la aparición de start-up en las universidades y en las instituciones académicas, a través de la creación de redes que ponen en contacto estos centros con agentes externos, tales como empresas o cámaras de comercio; mientras que el gobierno central (BMWi) establece los objetivos concretos del programa, los Länder lo implementan a través de actividades muy diversas. El programa Bioregio Contest se inició en 1997 con el objetivo de estimular la creación y competitividad de las empresas biotecnológicas y promover el establecimiento de compañías extranjeras en Alemania; los Länder ganadores del concurso reciben importantes fondos provenientes del BMBF que les permite, a su vez, atraer otra financiación pública y también privada tanto de Alemania como de países extranjeros.

Hasta aquí se han tratado los principales instrumentos de planificación que, junto con la Initiative for Excellence ya referida, conforman el conjunto de prioridades científicas en Alemania. Para completar este panorama hay que apuntar que en 2013 se presentó el National Research Infrastructure Roadmap, que pretende servir de apoyo y guiar las decisiones políticas en materia de infraestructuras, por ejemplo, en el momento de situar las grandes infraestructuras alemanas y también las europeas, pues este Roadmap enmarca en la estrategia de internacionalización del gobierno alemán y, concretamente, en su voluntad de apertura a la colaboración exterior. Junto a los instrumentos generales de planificación y del Roadmap se han puesto en marcha otros mecanismos específicos y sectoriales focalizados en cada una de las prioridades científicas; entre ellos destacan la Digital Germany 2015, el National Research Strategy BioEconomy 2030 y el Leading-Edge Cluster Competition.

\section{II.4. Colaboración científica público-privada y transferencia de conocimiento y resultados}

La promoción de la colaboración científica entre el sector público y el privado ha sido desde la década de los ochenta, una pieza muy relevante en la política científica estatal alemana. Los estrechos vínculos existentes entre el sector empresarial y la investigación pública se demuestran por el alto porcentaje de investigación en las universidades y en los pros que se lleva a cabo gracias a la financiación de la industria. En dirección inversa, el gobierno alemán ha puesto en marcha un amplio abanico de medidas de financiación pública para fortalecer la investigación en el sector empresarial; los programas temáticos son los más habituales en este

(16) Sobre ambos programas puede consultarse STAHLECK, T., BAIER, E., Analysis of the regional ..., cit., pp. 16-19. 
campo, que generalmente se orientan a conseguir objetivos concretos en determinados terrenos tecnológicos.

De entre los programas vigentes destaca el Industrial Collective Research for SMEs (IGF), que financia la investigación empresarial cooperativa organizada a través de la AIF, asociación de ámbito estatal que reúne a más de cien institutos de investigación en diferentes sectores y que se dedica especialmente a la promoción de las ciencias aplicadas en beneficio de las PYMEs. Las tareas de búsqueda se asignan a un instituto concreto y sus resultados se ponen a disposición de cualquier PYME del sector; de esta manera, el programa promueve la interacción entre los institutos de investigación y estos tipos de empresas.

En el terreno concreto de la transferencia, ya se han mencionado el programa EXIST, dirigido a la mejora de las condiciones para el emprendimiento en las universidades, y el Leading-Edge Cluster Competition, como una excelente fórmula de promover clusters de innovación prometedores; en idéntica dirección, en 2012 se puso en marcha el Zwanzig20Partnership for Innovation y 2013 también se abrió un nuevo Competence Centre for Procurers. Desde la perspectiva de la financiación, sobresale el High-Tech Start-up Fund y los programas del Federal Techology Venture Capital. En la misma línea, los gobiernos de los Länder ofrecen una serie de programas para las start-up relacionados con la innovación, que incluyen capital riesgo de características similares al que ofrece la Federación.

Si se focaliza la atención en las universidades, destacan tres instrumentos básicos de colaboración público-privada y de transferencia(17). El primero son las oficinas de transferencia de tecnología que establecen y refuerzan los contactos entre universidad y empresas privadas, aunque su efectividad es limitada por su escasez crónica de fuerza de trabajo. La segunda medida consiste en que recientemente las universidades ya pueden quedarse con las ganancias de sus contratos de investigación y usarlos para sus propios fines. Finalmente, el gobierno federal lanzó el Research Bonus, como incentivo temporal para la colaboración entre universidades y empresas, especialmente las PYMEs.

En suma, si se analiza el contenido de todos los instrumentos mencionados se deduce que la aproximación del gobierno federal en la colaboración y la transferencia científica ha sido triple(18): reducir los costes, incrementar los incentivos de las universidades y otros centros de investigación y aumentar los estímulos de las empresas a implicarse. En

(17) Sobre estos instrumentos puede consultarse KOSCHATZKY, K., STAHLECKER, T., The changing role of universities in the German research system: engagement in regional net-works, clusters and beyond. Fraunhofer, 2010.

(18) Así se explica en ROBIN, S. Y SHUBERT, T., Cooperation with public research institutions and success innovation: evidence form France and Germany. Fraunhofer, ISI, 2010, p. 6. 
esta triple dirección, existen múltiples programas de promoción de la colaboración público-privada y de la transferencia; además, hay reglas claras en materia de propiedad intelectual, no hay obstáculos para la colaboración y partenariados transnacionales y, comparativamente, la movilidad de investigadores entre instituciones públicas y privadas es sencilla. Sin embargo, tanto el gobierno como los expertos sostienen que hay un potencial desaprovechado en el campo de la traslación de los resultados a su comercialización y consideran conveniente la simplificación de la normativa y del considerablemente complejo sistema tributario, así como un mayor acceso a la financiación mediante fórmulas de capital riesgo y una mejora en la claridad de las medidas de apoyo a la l+D+i (19).

\section{Austria}

\section{III.1. Responsabilidades estatales en investigación e innovación}

En Austria la política científica se concibe como una competencia equiparable a la de fomento, por lo que puede ser desarrollada tanto por la Federación como por los nueve Länder (20); respecto de otras competencias que inciden en esta materia, la Constitución austriaca otorga facultades muy limitadas a los Länder en asuntos económicos y fiscales y reserva al Estado la competencia exclusiva en propiedad intelectual, asuntos relativos a la producción y a la industria, el régimen de patentes y el derecho laboral. En la práctica, el actor principal en política científica ha sido la Federación, aunque a partir de los años noventa los Länder comenzaron a implementar políticas propias con especial intensidad en el campo de la innovación; la excepción a esta tónica general se da con algunas regiones como la de Estiria, que tradicionalmente ha desplegado de forma destacada su competencia en investigación e innovación.

La coordinación en el campo de la ciencia entre Federación y Länder se produce principalmente a través de programas conjuntos, que se han iniciado fundamentalmente en la última década y que enfatizan el papel regional, pero donde el Estado desempeña un papel de liderazgo en todos los niveles, tanto de agenda como financiero. Buen ejemplo son los programas puestos en marcha por el gobierno federal y financiados tanto por éste como por los gobiernos regionales, donde la Federación

(19) De hecho, hay un buen número de programas e iniciativas encaminados a ofrecer información y acceso a la transferencia de tecnología; una síntesis de los mismos puede encontrarse en Voigt, P., ERAWATCH country reports 2013, cit., pp. 74-75.

(20) Sobre el sistema de distribución competencial austríaco se puede ver BUSSJÄGER, P., Homogeneidad y diferencia. Sobre la teoría de la distribución de competencias entre Bund y Länder en Austria. Instituto de Estudios Autonómicos, 2010. 
determina los objetivos así como las normas para su implementación; dos exitosos casos son el COMET-Competence Centers for Excellence Technologies, puesto en marcha en 2006, y la Academia+Business, puesto en funcionamiento en 2002 y reeditado en 2011 (21), a los que más tarde se hará referencia. Más allá de estos programas, la coordinación entre los dos niveles políticos es un punto débil del sistema, sin que haya un modelo claro de interacción entre los mismos. Precisamente, para mejorar la colaboración se creó en 2010 el Consejo de Investigación y Desarrollo Tecnológico (Plattform FTI Österreich), con representantes de ambos niveles y el objetivo de mejorar el flujo de información y coordinación. También a mitad de los años noventa la Federación y los Länder acordaron aumentar la coordinación en programas de investigación sobre medio ambiente y energía y en 2001 decidieron ampliar estos sectores, fundamentalmente, a las siete prioridades temáticas establecidas en el Sixth European Framework Programme(22). Por otra parte, las RIS3 han brindado la posibilidad de coordinación y de fijación de prioridades estratégicas entre la Federación y los Länder. Sólo tres Länder han formulado y registrado sus propias RIS3 (Viena, Alta Austria y Baja Austria) (23), aunque otros Länder como Estiria tienen desarrolladas sus propias estrategias de innovación y desarrollo económico. Ante esta situación, la Federación ha optado por formular una RIS3 de carácter nacional con capítulos regionales, lo que ha servido para fomentar la colaboración entre universidades, centros de investigación, agentes regionales y empresas, con el objetivo de que se conviertan en socios expertos en especialización inteligente. En todo caso, y en buena parte como consecuencia de la falta de una cooperación eficaz, la política de los Länder austriacos se construye a partir de las líneas maestras que establece la Federación(24), sin perjuicio de que los Länder vengan desarrollando una importante labor de asesoramiento a los agentes del sistema científico, especialmente en relación con el Programa Marco de Investigación y otros programas europeos de I+D+i, mediante los Puntos Regionales de Contacto (RCPS).

(21) Desde la perspectiva territorial, puede encontrarse más información sobre este programa en HABSBURG-LOTHRINGEN, Ci Ding, M., Analysis of the regional dimensiones of investment in research. Case study regional report: Carinthia (Germany). ERAWATCH, 2006, p. 13.

(22) Sobre estas iniciativas de coordinación sectorial se puede ver KAISER, R. y PRANGE, H., "Managing diversity in a system of multi-level governance: the open method of coordinación in innovation policy». Journal of European Public Policy, núm. 11: 2, 2004, p. 256.

(23) Sobre el contenido de las RIS3 regionales austriacas puede verse ERAWATCH Country Reports 2013: Austria. European Commission, 2014, pp. 20-22.

(24) Así ho afirma JÖRG, L., Policy profile Austria. TIP Input papel for the OECD NIS MONITOR Network, work package 1. Technopolis, 2004, p. 12. 


\section{III.2. La gobernanza de la ciencia}

Los principales órganos que dirigen la política científica en Austria se encuentran en el nivel estatal(25). Hasta la formación del último gobierno tras las elecciones generales de septiembre de 2013, los Ministerios responsables eran el Ministerio de Ciencia e investigación (BMWF), el de Economía, Familia y Juventud (BMWFJ) y el de Transporte, Innovación y Tecnología (BMVIT). Ahora los dos primeros se han unido en el nuevo Ministerio de Ciencia, Investigación y Economía (BMWFW), que acumula competencias en educación superior (universidades, universidades de ciencias aplicadas y formación profesional superior); en investigación básica; en política económica; en innovación y desarrollo tecnológico; y en promoción del emprendimiento. Dependen del BMWFW instituciones no universitarias de investigación, como el Institute of Science and Technology (IST Austria), la Austrian Academy of Sciences y la Ludwig Boltzman Society (LBG). También depende de este Ministerio la Austrian Science Fund (FWF), principal órgano de financiación de la investigación básica. Igualmente participa en el $50 \%$ de la Austrian Business Service (AWS) y en el Austrian Research Promotion Agency (FFG), y apoya a los centros tecnológicos, los Austrian Cooperative Research Institutes (ACR-Institutes) y a la Christian Doppler Research Association (CDG, de la que dependen los CD-Labs).

Esta fusión ministerial ha sido objeto de algunas críticas, que consideran que la misma puede limitar la libertad científica y la independencia de los grupos de investigación básica, además de reducir la inversión en este ámbito; por el contrario, refuerza la inversión en investigación aplicada y transferencia del conocimiento, aumentando así la alineación de la política científica con las políticas industriales del país para, finalmente, favorecer el desarrollo de la I+D+i en la empresa. Estos temores están presentes en ese momento en todos los países europeos, ya que la necesidad de vincular el sector productivo al conocimiento puede llevar a desequilibrar el apoyo a la investigación básica (26). No obstante, hay que señalar que, con anterioridad, la existencia de los dos Ministerios había sido también criticada en Austria, ya que dificultaba la unidad de acción y era considerada una debilidad del sistema de gobernanza. Precisamente, y como se explicará, una de las prioridades de la vigente Austrian Federal Government Strategy for Research 2011 es tratar de aumentar la coordinación intergubernamental.

(25) Un cuadro explicativo de todos los agentes implicados en la gobernanza científica austríaca puede encontrarse en CUNTZ, A., ERAWATCH country reports 2012: Austria. European Commission, 2013, p. 7.

(26) En cuanto al caso austriaco puede ver Austrian Research and Technology Report 2014. BMW y BMVIT, 2014, p. 49. 
Tal y como se ha adelantado, el último ministerio competente en I+D+i es el BMVIT, responsable de las ciencias aplicadas, de la transferencia tecnológica y con participación en el otro $50 \%$ de la AWS y del FFG, además de ser accionista mayoritario del Austrian Institute of Technology (AIT). Sin embargo, a pesar de no ser directamente competente en política científica, el Ministerio de Finanzas (BMF) juega también un papel fundamental en la materia, ya que determina la asignación de recursos económicos y también el diseño, la implementación y el monitoreo de los programas que elaboran el BMWFW y el BMVIT conjuntamente: los Structural Programmes, que financian proyectos ejecutados por las empresas; y los Thematic Programmes, que promueven los proyectos entre las empresas y las organizaciones científicas en el marco de las prioridades temáticas nacionales. También depende del BMF del Austrian Institute of Economic Research (WIFO) y el Institute for Advanced Studies (IHS). A nivel consultivo, en Austria hay dos órganos: el Austrian Science Council, que asesora al gobierno federal en temas relacionados con la universidad; y el Austrian Council for Research and Technological Development, que asesora a los gobiernos federal y de los Länder en política científica, especialmente en materia de gobernanza, además de definir la política científica estatal y monitorear su implementación (27).

Para gestionar la política científica a nivel federal hay tres agencias que ya se han mencionado: la FFG, el FWF y el AWS. La FFG es responsable de la promoción científica y tecnológica de la industria, de la orientación temática de la ciencia (por ejemplo, en nanotecnología, TIC, etc.), de la cooperación entre la ciencia y la industria, los programas europeos y del programa nacional espacial; además, es responsable de los programas más importantes desde el punto de vista de su impacto en los Länder, como son los Structural Programmes de la FFG-Bassis Programm. El FWF es el principal gestor de la promoción de la investigación básica, que se focaliza en las instituciones de educación superior; así, el $70 \%$ de sus fondos se destinan a proyectos competitivos orientados en el ámbito de este ciclo formativo. Finalmente, el AWS se describe a sí mismo como el «banco especial para el apoyo a las empresas» pues les ofrece préstamos, créditos, garantías y seguros, así como servicios de consultoría y contactos con business angels; concretamente, juega un papel primordial en la financiación y apoyo a las start-up. A nivel de implementación de la política científica, en Austria hay 22 universidades públicas, 21 de ciencias aplicadas y 12 universidades privadas de tamaño reducido. Las primeras son la columna vertebral de la investigación básica y las segundas

(27) La relevancia este órgano como asesor en materia de gobernanza científica explica en HARTMAN, C. y BERGER, M., Analysis of the regional dimensiones of investment in research. Case study regional report: Styria (Austria). ERAWATCH, 2006, p. 15. 
se establecieron para diversificar la enseñanza universitaria y encadenarse a las demandas del mercado de trabajo, por lo que su tarea principal es proveer una oferta de educación superior desde una vertiente práctica (28).

\section{III.3. La planificación de la política científica}

La planificación de la ciencia en Austria se encuentra recogida en la Austrian Federal Government Strategy for Research 2011: Realising potentials, increasing dynamics, creating the future: Becoming an Innovation Leader. Esta Estrategia supone la superación de dos de las más relevantes debilidades estructurales de la política científica austríaca: disponer de una política clara a largo plazo que contara con el apoyo de los agentes implicados en el sistema de la ciencia. Así, en este plan se recoge una largamente esperada planificación de la política científica, compartida por los diferentes ministerios y en la concepción de la que han participado los principales agentes del sistema(29). Desde la perspectiva de su contenido, la Estrategia también supone un paso adelante. A pesar de que Austria se ha caracterizado tradicionalmente por una política científica genérica, este plan defiende que una aproximación no selectiva resulta insostenible $y$, por lo tanto, es necesario focalizar los instrumentos de financiación (30). En esta dirección, además de girar la política hacia el sector industrial productivo, la Estrategia identifica como grandes prioridades temáticas, el cambio climático, los recursos escasos, la calidad de vida y el cambio demográfico. Para lograr estas prioridades, las principales tareas gubernamentales pasan por fortalecer las estructuras estatales de investigación, con el foco puesto en la excelencia; promover las capacidades innovadoras de las empresas; mejorar la gobernanza; y unir la I+D+i al sistema educativo.

Desde el punto de vista de su implementación, resultan interesantes dos cuestiones de distinta naturaleza pero claramente conectadas. En primer lugar, la puesta en funcionamiento tres meses después de ser aprobada la Estrategia de la llamada Task Force, liderada por la Cancillería y por el Ministerio de Finanzas y donde participan delegados de los Minis-

(28) Sobre la gobernanza en las universidades austriacas puede leerse, DE BOER, H., ENDERS, J. y SCHIMANK, U., "¿Hacia una nueva gestión pública? ...», Cit., Pp. 193-214.

(29) Sobre estas críticas y sobre cómo se ha desvanecido tras la aprobación de la Estrategia de 2011 se puede leer Schuch, K., ERAWATCH country reports 2011: Austria. European Commission, 2012, p. 13.

(30) Este cambio hacia la priorización temática en Austria se explica en IZSAK, K. y GRINIECE, E., Innovation policy ..., cit., Pp. 13-14. 
terios implicados en política científica; su principal función es dirigir y asegurar la ejecución coordinada de la Estrategia por parte de todos los departamentos, por lo que se crearon nueve grupos de trabajo interministeriales en los ámbitos considerados claves dentro de la Estrategia (31). En segundo lugar, como consecuencia de la puesta en marcha del plan se produjeron cambios organizativos a nivel ministerial, con la intención de evitar problemas en la aplicación de la Estrategia derivados de la anterior confrontación entre los diferentes ministerios.

Atendiendo a las actuaciones sectoriales, la fuga de cerebros que afecta a todos los países europeos también preocupa en Austria y se han puesto en marcha diferentes programas a nivel federal, como el Brainpower Austria Programme o el Carreer Grants Programme. También son destacables las políticas de igualdad de género que se han ido adoptando para paliar la importante brecha de género existente en el ámbito de la I+D+i, pues en 2.013 Austria ocupaba la decimonovena posición en el ranking mundial en cuanto a desigualdad hombre/mujer: para empezar, la reforma de la Ley de Universidades de 2009 obligó a respetar una cuota del $40 \%$ de mujeres en todos los órganos y comisiones de la universidad; además, los acuerdos de financiación de las universidades contemplan entre los criterios de dotación el cumplimiento de indicadores de género; finalmente, desde 2002 el programa Women in Research and Technology incluye varias acciones en el ámbito académico, de la investigación aplicada y del sector educativo preuniversitario.

\section{III.4. Colaboración científica público-privada y de la transferencia de conocimiento y resultados}

Desde la década de los noventa, la colaboración entre universidades, organizaciones de investigación y sector privado está apoyada en Austria por un amplio abanico de estructuras. Entre ellas destacan los aludidos COMET-Competence Centers for Excellence Technologies, con programas a medio-largo plazo co-financiados por Federación y Länder y concertados entre científicos e industria, que centran su investigación en las aplicaciones industriales. También sobresale la FFG, que ofrece hasta el $70 \%$ de financiación a las start-up en proyectos tecnológicamente arriesgados pero económicamente prometedores, además de apoyar a las empresas jóvenes en la búsqueda de partners o inversores potenciales a través de

(31) Sobre la concreta composición de la Task Force y su trabajo se puede consultar Schuch, K., ERAWATCH country reports ..., cit., pp. 14-15, y Austrian Research and Technology Report 2014, Federal Ministry of Science and Research \& Federal Ministry for Transporte, Innovation and Technology, 2014. 
foros. Entre estas estructuras también se encuentran los CD-Labs, la LBG y el AIT.

Con carácter más reciente, el gobierno austriaco ha desarrollado un exitoso espectro de programas. En la última década estos se han dedicado a apoyar la creación de empresas de tecnología e innovación, que ahora se encuentran bajo el paraguas de la Austrian Association of Technology Centers (VTO). También se han iniciado programas para apoyar el emprendimiento, como el ya mencionado Academia+Business, que apoya económicamente la creación de incubadoras en las universidades e instituciones de investigación (AplusB-Centros). Entre las últimas actuaciones puestas en marcha por el gobierno austriaco se encuentra la promoción de capital riesgo privado, especialmente destinado a las jóvenes PYMEs innovadoras en sus primeras fases de funcionamiento; en este contexto, el programa más importante es el Venture Capital/Clean-Tech Initiative. Un nuevo programa reseñable es el KLIPHA, cuyo objeto es financiar los estudios experimentales de proyectos innovadores de las PYMEs; la financiación se facilita a través de préstamos, pero en determinadas circunstancias se transforma en una subvención si al final los resultados de los estudios son negativos. Otro reciente mecanismo es el Innovation Voucher, que trata de ayudar a las PYMEs que se inician en la actividad innovadora; este ha sido uno de los programas que más éxito ha tenido en los últimos años, financiando hasta 492 proyectos en 2013(32). Ese mismo año se puso en marcha también el Frontrunners Programme, destinado a apoyar a las empresas claves en el posicionamiento de Austria a nivel internacional como país líder en innovación, con un programa de ayudas para las empresas con mayor esfuerzo innovador. Ya en 2014, el BMWFW inició un nuevo programa, el Knowledge Transfer Centers and Exploitation of IPR (Intellectual Property Rights), con tres módulos dirigidos a incentivar las relaciones entre universidades $y$ empresas; estimular las patentes; y acelerar la explotación de las invenciones creadas en las universidades. Finalmente, también el 2014 se ha creado un centro especializado en ciencias de la vida y tres centros regionales, que han supuesto un revulsivo a nivel territorial para incrementar las relaciones entre la universidad y los organismos de investigación con el entorno productivo(33). Desde la perspectiva sectorial, el 2013 se ha creado un nuevo consorcio de investigación en el ámbito de las tecnologías verdes, el Green Tech Research. Esta nueva red reúne cinco instituciones de investigación, incluyendo el FH Joanneum y socios de negocio,

(32) Así se explica en Austrian Research and Technology Report 2014, cit., P. 99.

(33) Sobre el impacto de estos centros puede consultarse CUNTZ, A., ERAWATCH country reports 2014: Austria European Commission, p. 43; y a Austrian Research and Technology Report 2014, cit., p.11. 
con el objetivo de poner en común sus conocimientos en el campo de la energía y las tecnologías medioambientales.

Como se desprende de la gran cantidad de estructuras y programas existentes, el sistema federal de promoción de la colaboración y la transferencia científica es muy complejo en términos de gobernanza y se encuentra fragmentado, con prevalencia de cuantiosas intervenciones de reducida escala. Además, aunque el $35 \%$ de las grandes empresas austriacas colaboren con las universidades, la cooperación entre las PYMEs y la universidad es muy baja; precisamente para promover la colaboración científica entre la academia y las PYMEs que no tienen unidades de investigación se creó el programa Young Experts, que financia el $80 \%$ del coste laboral del puesto de trabajo del post-doc contratado por la empresa.

\section{Reino Unido}

\section{IV.1. Responsabilidades estatales en investigación e innovación}

Comparado con otros países, el diseño político del Reino Unido está menos determinado por unos marcos legislativos rígidos(34). Así, para dar cuenta de los mecanismos que rigen el sistema de I+D+i es necesario recurrir tanto a instrumentos de tipo legislativo, como las Acts, como otros de carácter más informal, pero que marcan también la práctica del sistema de investigación. Teniendo en cuenta este entorno normativo más débil, destacan entre los principios y normas que rigen las competencias y la distribución de los recursos de I+D+i, el Haldane Principle, los Royal Charters y la Science and Tecnology Act 1965 (STA) (35).

El Haldane Principle determina que es la comunidad científica la mejor capacitada para proponer y seleccionar los proyectos de investigación, sobre la base del mérito científico. Dentro de este marco, corresponde al gobierno establecer las grandes prioridades estratégicas y los fondos disponibles para distribuir entre los diferentes Research Councils (RC), mientras que la comunidad científica se encarga, en coordinación con los mismos, de seleccionar los proyectos. De esta manera, se establece un

(34) En cuanto al concreto ámbito de la I+D+i puede verse Simmonds, P., Rosemberg MONTES, C., SHARP, T., RENTEL, M. y Wain, M., Coordination and governance of the UK science, technology and innovation (STI) system between the national and sub-national level. Technopolis, 2014.

(35) El Haldane principle recibe su nombre de Richard Burdon Haldane, político liberal, abogado e intelectual que fue Lord Chancellor dos veces y precursor de esta línea política a principios de siglo $x x$. Puede encontrarse información sobre estos instrumentos en EDGERTON, D., «The Haldane Principle and other invented traditions in science policy». History and Policy, www.historyandpolicy. org/papers/policy-paper-88. 
procedimiento de "abajo hacia arriba" por el que se eligen los proyectos en base a las diferentes líneas de desarrollo científico. Muchas instituciones del sistema de I+D+i del Reino Unido operan bajo los Royal Charters, o Cartas de Derechos, que otorgan entidad legal a una colectividad de individuos y requieren la aprobación del gobierno; además, ser una entidad sujeta a Carta de Derechos tiene implicaciones sobre su supervisión interna, pues obliga a otorgar parte del control al Privy Council. Los siete $\mathrm{RC}$ operan bajo Royal Charters, lo mismo que la mayor parte de las universidades del país. Sin embargo, actualmente existen nuevas figuras jurídicas para otorgar derechos, como hizo la Further and Higher Education Act 1992 respecto de la financiación y administración de la educación superior en Inglaterra y Gales y con efectos en Escocia (36). La STA puede considerarse como la herramienta legislativa más destacada en la articulación del sistema de I+D+i del Reino Unido, donde se establecen las responsabilidades en búsqueda de la Secretaría de Estado, el Ministerio, las entidades con estatus de Royal charters y otras organizaciones. Además, contiene previsiones relativas a los RC ya la distribución de actividades relacionadas con la investigación.

\section{IV.2. La gobernanza de la ciencia}

El Reino Unido tiene un sistema de I+D+i complejo en el que se incluyen diferentes agentes de financiación y ejecución, públicos y privados(37). En este contexto, la gobernanza de la I+D+i tiene como máximo responsable al gobierno central, que establece la dirección y las prioridades generales de la investigación científica; una vez establecidas las directrices principales, los diferentes ministerios coordinan y ejecutan políticas sectoriales, donde destaca el Department for Business Innovation and Skills (BIS). En un nivel operativo, se encuentran las diferentes agencias de financiación, con entidades como los RC. Desde el punto de vista de la implementación, diversos organismos públicos y privados se encargan de la ejecución de la investigación, como las universidades, que gozan de un nivel de autonomía considerable. A continuación se detallan las competencias de cada uno de estos niveles de gobernanza.

(36) Esta Ley destaca por haber otorgado la potestad a 35 politécnicos para convertirse en universidades y por la creación de entidades de financiación como el Higher Education Funding Council for England (HEFCE) y el Further Education Funding Council (FEFCO), así como entidades similares en Escocia y Gales que hasta entonces eran financiadas por medio de una única entidad (Universities Funding Council) para todo el Reino Unido. También eliminó el control de los gobiernos locales sobre los colegios de educación y creó acuerdos para el control de calidad.

(37) Una exposición del mismo puede encontrarse en CUNNINGHAM, P., ERAWATCH country reports 2013: United Kingdom. European Commission, 2014. 
Empezando por el nivel más elevado, tal y como se acaba de exponer, el BIS es el ministerio que tiene la responsabilidad general en ciencia e innovación a nivel nacional y también posee competencias en educación superior, lo que le otorga una gran capacidad de acción global en temas de I+D+i. Concretamente, goza de poderes ejecutivos en el ámbito de la investigación, se encarga de la elaboración y seguimiento de la estrategia de investigación y es el proveedor principal de recursos públicos para la investigación científica. También provee de fondos a los RC, que los canalizan a las instituciones de educación superior y los Research Council Institutes.

EI BIS trabaja con un total de 51 agencias y entes públicos, que se encargan del desarrollo y coordinación de las políticas y tienen funciones regulatorias. Estos organismos incluyen, entre otros, Departamentos no ministeriales, Agencias ejecutivas y Entes Públicos no departamentales. Los Departamentos no ministeriales tratan asuntos donde la supervisión política se considera no apropiada o podría dar lugar a injerencias políticas; suelen tener funciones regulatorias o supervisoras y están encabezados por funcionarios públicos. Los Entes Públicos no departamentales no forman parte del gobierno y rinden cuenta ante el Parlamento, lo que les otorga mayor independencia; también difieren de las Agencias ejecutivas en que no llevan a cabo políticas ministeriales y tienen, de esta manera también, una mayor autonomía (38).

Los RC se distribuyen en siete disciplinas (39) y se coordinan de forma asociativa mediante el Research Councils UK (RCUK). A su vez, el Joint Strategy Group (JSG) se encarga de facilitar la coordinación en el tratamiento de asuntos estratégicos entre el RCUK y el BIS y de proveer consejo al Director general de conocimiento e innovación, al igual que el Council for Science and Technology aconseja al Primer Ministro ya los Primeros Ministros de Escocia y Gales en temas estratégicos (40). Además de los siete RC, Reino Unido también cuenta con cuatro entes de financiación que se encargan de repartir fondos, directas o institucionales, en las universidades (41): el Higher Education Funding Council for England (HEFCE), el Scottish Funding Council (SFC) y el Higher Education Funding Council

(38) Se puede encontrar una relación de las agencias y organismos que trabajan con el BIS en https://www.gov.uk/government/organisations\#departments [Fecha acceso: 03/06/2015].

(39) Enlaces de los differents Research Councils: AHRC; BBSRC; EPSRC; ESRC; MRC; NERC; STFC [Fecha acceso 03.06.2015].

(40) Sobre las funciones del Consejo puede consultarse Jasanoff, S., "Quality control and peer review in advisory science». A: Lentsch, J. y Weingarten, P. (comp.), The Politics of Scientific Advice: Institutional Design for Quality Assurance. Cambridge University Press, 2011, pp. 19-35.

(41) Sobre esta financiación miedo verse https://www.gov.uk/government/policies/investing-inresearch-development-and-innovation/ supporting-pages / science and research-funding [Fecha de acceso: 10:03. 2015]. 
for Wales (HEFCW); en Irlanda del Norte la financiación lo tramita directamente el Department for Employment and Learning (DELNI). Desde la perspectiva de la financiación, también hay que destacar el Technology Strategy Board (TSB), que focaliza su actividad en el fomento de la transferencia de conocimientos y resultados y al que posteriormente se aludirá.

La ejecución de la investigación va principalmente a cargo de las instituciones de educación superior, los Organismos Públicos de Investigación (OPIs) y el sector empresarial, a los que seguidamente se hace referencia. En 2012, se contabilizaron un total de 165 instituciones de educación superior, de las cuales la mayor parte son universidades. Las universidades británicas gozan de gran autonomía para la búsqueda y la gestión de fondos, provocando que se encuentren entre las universidades europeas con portafolios de financiación más diversificados (42); sin embargo, la mayor parte de los fondos son públicos, ya sean directos o indirectos (43). El sistema de educación superior varía enormemente en cuanto a su actividad de investigación, con universidades con una alta actividad investigadora de reconocimiento mundial y otros con una orientación más centrada en la enseñanza (44). Por otra parte, alrededor de unos 100 OPIs desarrollan investigación propia y tienen capacidad para financiar investigación con una orientación aplicada, siempre que se considere relevante para las entidades de las que dependen (que van del Museo Británico en el Laboratorio de seguridad). El proceso de transformación del sistema de la ciencia en el Reino Unido también ha afectado a estas instituciones, con una tendencia a la privatización desde los años setenta(45). Finalmente, el Reino Unido cuenta con un tejido empresarial activamente involucrado en actividades de I+D+i. Sin embargo, el gasto del sector privado es bajo si se compara con países como Francia, Estados Unidos, Dinamarca, Japón o Suecia (46), y en este aspecto el país se considera un follower en innovación según el IUS 2014.

(42) Así se explica a de Dominic, L., PÉREZ, SE y FERNÁNDEZ-ZUBIETA, A., European university funding and financial autonomy. A study on the degree of diversification of university budget and the share of competitive funding. Publications Office of the European Union, 2011.

(43) En relación con esta financiación público se puede consultar Geun, A., «The changing rationale for European university research funding: are there negative Unintended consequences?», Journal of Economic Issues, 2001, pp. 607-632; y Geun, A. y MARTIN, BR, "University research evaluation and funding: An international comparison», Minerva, num. 41, 4, 2003, pp. 277 a 304.

(44) Reino Unido cuenta con ocho universidades dentro de las 100 mejores del Shanghai Ranking 2014: http://www.shanghairanking.com/es/World-University-Rankings-2014/UK.html [Fecha acceso: 03/10/2015].

(45) Al respecto puede verse BODEN, R., COX, D., NEDEVA, Mi BARKER, K., Scrutinising science: The changing UK government of science. Palgrave-Macmillan, 2004.

(46) En relación con estos datos puede consultarse CUNNINGHAM, P., ERAWATCH country reports 2013: United Kingdom ..., cit ; y HUGHES, A. y MINA, A., The UK R \& D Landscape. Enhancing Value Task Force, UK-IRC, 2012. 


\section{IV.3. La planificación de la política científica}

La Innovation and Research Strategy for Growth 2011 (IRS) establece el marco estratégico gubernamental en investigación e innovación. Esta Estrategia se complementa con la UK Industrial Strategy 2012, que identifica los sectores más relevantes para el crecimiento de la economía del Reino Unido. Finalmente, los gastos gubernamentales se plasman en los Spending Reviews anuales. Seguidamente se analizarán cada uno de estos documentos.

La IRS parte de la necesidad de mantener una investigación básica de excelencia, al tiempo que considera la innovación como clave para competir en la nueva economía global. En este marco, reconoce el papel central del sector privado en la innovación pero sin menospreciar el rol que el gobierno debe jugar a través de la financiación de la investigación básica; de la mejora de las relaciones entre las instituciones de educación superior y el sector privado; y del fomento de un entorno más propicio para la comercialización de la investigación. Del mismo modo, la IRS admite que el gobierno puede hacer un mayor uso de la compra pública innovadora y mejorar la financiación de otras iniciativas en este ámbito, como la Small Business Research Initiative; también admite la importancia de facilitar el acceso a los datos y al conocimiento financiado por fondos públicos y de mejorar la regulación de la propiedad intelectual. Desde el punto de vista del contenido, la estrategia acepta la exigencia de ajustarse a los límites del déficit público y, por tanto, reivindica la necesidad de priorizar determinadas áreas; en este contexto, la IRS prioriza los retos como el cambio climático, la seguridad o la demografía, y destaca el papel que pueden tener sectores como la gestión de big data para solucionar estos grandes retos. Entre los sectores tecnológicos claves, la IRS prevé cuatro: las ciencias naturales en áreas como la biotecnología, donde destacan la UK Life Sciences Strategy y el Biomedical Catalyst Fund; la producción en sectores de alto valor añadido, como la aeronáutica, donde sobresale el programa NovaSar que desarrolla radares para satélites; las tecnologías digitales, relacionadas por ejemplo con las industrias creativas; y la nanotecnología. En la Estrategia también se señalan cuatro tecnologías emergentes donde hay que priorizar el gasto: biología sintética; cómputo de eficiencia energética; acumulación de energía; y el grafeno, por medio del Graphene Global Research and Technology Hub.

Como se ha adelantado, la UK Industrial Strategy 2012 complementa la IRS y establece 11 sectores para los que se elaboran estrategias de desarrollo específicas, en colaboración con el sector empresarial. Estos sectores son el aeroespacial; las tecnologías de la agricultura; la automoción; la construcción; la economía de la información; la educación internacio- 
nal; las ciencias naturales; el sector nuclear; la energía eólica fuera de costas; el gas y el petróleo; y los servicios profesionales de negocios. La Estrategia industrial también establece una lista de 8 grandes tecnologías en las que el gobierno, junto con el RCUK y el TSB, consideraron que el país puede tener una ventaja competitiva: el big data y la computación de eficiencia energética; los satélites y aplicaciones comerciales del espacio; la robótica y sistemas autónomos; las ciencias naturales, genómica y biología sintética; la medicina regenerativa; la agro-ciencia; los materiales avanzados y la nano-tecnología; y la energía y su acumulación. Finalmente, el Spending Review 2013 establece la distribución de gasto del gobierno entre abril de 2015 y de 2016 y la forma en que se financian servicios prioritarios como los de salud, educación e infraestructuras. Es liderado por el Ministro de Economía y hacienda, que en el caso de la investigación negocia con el BIS gasto total en $\mathrm{I}+\mathrm{D}+\mathrm{i}(47)$. Adicionalmente, hay que apuntar que otros documentos como el Annual Innovation Report permiten evaluar la evolución de las estrategias en I+D+i y se inscriben en la tendencia progresiva de utilizar indicadores en las decisiones gubernamentales y departamentales (48). Este documento ofrece evidencias sobre los retos de innovación identificados e incluye nuevas medidas y desarrollos.

\section{IV.4. Colaboración científica público-privada y transferencia de conocimiento y resultado}

En las políticas de cooperación científica público-privada y de transferencia de conocimiento y resultados destacan el papel del TBS y los Technology and Innovation Centros o catapulta, así como la labor de los diferentes RC y del HEFCE. EITSB, o Innovate UK, financia la investigación aplicada para facilitar el proceso de comercialización de productos. Para optar a su financiación se requiere a los proyectos que estén liderados por empresas o actúen en colaboración con universidades. En su documento estratégico sobre innovación, Ilamado Concept to Commercialisation: a strategy for business innovation 2011-15, el TSB pretende: (i) acelerar el viaje entre el concepto y la comercialización, mediante el apoyo a empresas pequeñas y en estado inicial, la creación de redes de tecnología y centros de innovación, la promoción de intercambio de conocimiento y la innovación abierta; (ii)

(47) Sobre las cantidades asignadas a IDE en los Spending Reviews puede leerse Simmonds, P. y otros, Coordinación and governance of the UK, cit.

(48) En este sentido puede consultarse MARTIN, BR, «The use of multiple indicators in the assessment of basic research». Scientometrics, núm. 36, 3, 1996, pp. 343-362. 
conectar el entorno de la innovación, mediante la conexión de las organizaciones involucradas en las actividades innovadoras, desde los agentes de financiación a los ejecutores; (iii) convertir la acción del gobierno en oportunidades de negocio, mediante la identificación de áreas en que la política puede estimular el negocio innovador; (iv) invertir en las áreas prioritarias de la política industrial; (v) y aumentar las capacidades delTSB en paralelo a la instauración de un proceso de rendimiento de cuentas y monitoreo de la institución. En este contexto, el TSB se configura como la principal agencia de innovación del Reino Unido, que desarrolla su labor a través de una variedad de instrumentos entre los que se encuentran: TSB direct communications, TSB technologies, Knowledge Transfer Networks, knowledge Transfer Partnerships, Granst for Collaborative Research, Innovation vouchers y los Puntos de Contacto Nacional para el Programa Marco de la UE. Sin embargo, dentro de estos instrumentos destacan las catapultas, centros tecnológicos e innovadores especializados en las áreas identificadas en la UK Industrial Strategy 2012.

Las catapultas han sido diseñadas para cubrir el hueco entre las empresas, la academia, la investigación y el gobierno, dando apoyo a los proyectos y tecnologías que están listos para su testeo pero que no están suficientemente maduros para entrar en el mercado. Pretenden garantizar que estas ideas evolucionen hasta su comercialización, focalizándose en los potenciales huecos existentes entre la financiación y los incentivos económicos de posibles inversores, pues se trata de proyectos donde los agentes privados, como los business angels (49), están menos dispuestos a invertir por el alto riesgo de fracaso. Las catapultas consiguen sus fondos de una manera variada, combinando la financiación competitiva, el privado y los fondos propios delTSB.

En cuanto a los RC, disponen de "rutas" para la transferencia de conocimiento y resultados que se enmarcan dentro de sus planes estratégicos (50). Las "rutas" incluyen becas de investigación supervisadas de manera conjunta entre entidades públicas y privadas, mediante los Cooperative Awards for Science and Engineering (51). Además, en el RCUK existe un grupo de trabajo específico sobre innovación e impacto no académico de la investigación, como un medio para coordinar las

(49) Los business angels son inversores que ofrecen capital inicial para las empresas de nueva creación (start-ups) que se encuentra $\mathrm{n}$ en estadios iniciales de desarrollo. OECD, "Financing highgrowth firms: The role of angel investors», OECD, 2011.

(50) Para ver links a las «rutas» de cada RC puede consultarse http://www.rcuk.ac.uk/ke/impacts/ [Fecha acceso: 04/09/2015].

(51) Sobre estas becas se puede ver Cunningham, P., ERAWATCH country reports 2013: United Kingdom, cit. 
actividades entre los diferentes $\mathrm{RC}$ y facilitar el aprendizaje (52). Finalmente, el HEFCE también tiene un papel importante en la transferencia de conocimiento y resultados por medio del Higher Education Innovation Fund (Heifer). Se trata de un fondo nacional para el apoyo del desarrollo institucional de políticas, estructuras y capacidades para la transferencia del conocimiento, que cuenta con más de dos décadas de experiencia (53).

\section{España}

\section{V.1. Responsabilidades estatales en investigación e innovación}

La distribución de competencias entre Estado y comunidades autónomas (CCAA) en investigación y desarrollo responde a un criterio que no resulta habitual en el sistema de reparto competencial establecido en la Constitución Española (CE). Si bien la tónica general consiste en reservar en exclusiva competencias a una de estas instancias o asignar diversas funciones sobre una misma materia, en este caso la Constitución establece que tanto el Estado como las comunidades son competentes en el fomento de la investigación científica y técnica y añade que será aquél el encargado de la coordinación general en esta materia (artículos 149.1.15 y 148.1.17 CE). De esta forma, ambas instancias disponen de funciones legislativas y ejecutivas plenas produciéndose una concurrencia total en este ámbito, bajo la obligada coordinación estatal (54).

El Tribunal Constitucional (TC) ha tenido oportunidad de pronunciarse en diversas ocasiones en relación con esta distribución de competencias, por lo que ha realizado puntualizaciones que arrojan luz sobre este particular reparto. Para comenzar, el Tribunal ha delimitado la competencia de fomento indicando que se extiende a cualquier materia (industria, agricultura, etc.) con independencia de quién disponga de competencias sobre la misma; además, el fomento abarca tanto las actividades directamente conducentes a descubrimientos científicos como

(52) En relación con este grupo de trabajo puede verse SIMMONDS, P. y otros, Coordination and governance of the UK, cit.

(53) Respecto de las funciones del HEFCE puede verse http://www.hefce.ac.uk/whatwedo/kes/heif/. En esta página también se encuentra el informe sobre la labor de esta institución Strengthening the Contribution of English Higher Education Institutions to the Innovation System: knowledge exchange and Heifer funding (2012) [Fecha acceso: 03/06/2015].

(54) Un análisis exhaustivo sobre el reparto competencial en política científica entre Estado, CCAA y entes locales puede encontrarse en GÓMEZ, M., "Administración e investigación científica y técnica: veinte años después de la Ley de la Ciencia». Revista de Estudios autonómicos y Federales, núm. 5, 2007, pp. 241-270. 
la divulgación de los resultados obtenidos (STC 53/1988). La jurisprudencia constitucional ha concretado igualmente que estas actividades incluyen, no sólo el apoyo a la investigación privada o universitaria, sino también la creación de una estructura de investigación propia, estatal o autonómica (STC 90/1992) (55).

Otro aspecto relevante tratado por el TC ha sido la delimitación de la capacidad estatal para coordinar la investigación científica y técnica, que viene justificada por la plena concurrencia de competencias en la materia. En este punto el Tribunal ha aplicado su conocida doctrina relativa a la coordinación estatal, que parte del equilibrio entre poner en marcha las medidas necesarias y suficientes para lograr la integración de la diversidad en el conjunto del sistema y evitar que la concreción y desarrollo de las mismas vacíe las competencias autonómicas, en este caso, en fomento de la investigación (STC 90/1992). Se hace evidente aquí el amplio margen de apreciación de que dispone el Estado en el uso de su poder de coordinación, que también podrá ser de carácter preventivo.

Finalmente, elTC ha defendido la capacidad del Estado para establecer partidas presupuestarias con destino a transferencias de diverso tipo, en desarrollo de su competencia de fomento de la investigación (STC 13/1992). Además, dispone de una plena potestad de gasto, de manera que son posibles las subvenciones estatales que regulen las condiciones de otorgamiento de recursos financieros e, incluso, la tramitación y resolución de los expedientes de solicitud; en definitiva, puede producirse una gestión totalmente centralizada de las subvenciones estatales dirigidas al fomento de la investigación (STC 186/1999 y 175/2003) (56). En este punto conviene aclarar que en investigación y desarrollo no se han producido traspasos de medios del Estado a las CCAA, pues aquél mantiene la competencia al igual que la desarrollan las comunidades (STC 90/1992). Todo ello no excluye, por supuesto, que la capacidad de gasto del Estado se articule a través de convenios con las CCAA.

El caso de la innovación, en cambio, queda relativamente al margen de este escenario de distribución competencial. Dado que el artículo 149.1 CE no prevé expresamente la competencia estatal en innovación, los Estatutos de autonomía pueden asumir de forma plena la competencia sobre esta materia. Ello supone que el campo de actuación de las comunidades en este ámbito es superior del que disponen en ciencia y

(55) Una explicación detallada sobre estos aspectos del reparto competencial en materia de fomento de la investigación científica y técnica puede hallarse en CUETO, M., Régimen jurídico de la investigación científica: la labor investigadora de la Universidad. Ed. Cedecs, 2002; y FONSECA FERRANDIS, F., Estado, CCAA y ciencias biomédicas; Hacia un modelo de cohesionar. Ed. Thomson Civitas, 2007.

(56) El ejercicio de la potestad subvencional de gasto público como medida de fomento se trata ampliamente en CUETO, M., Régimen jurídico..., cit., pp. 238-262. 
tecnología, pues no se encuentran sometidas a la coordinación general del Estado. Esta disociación también ha tenido su reflejo en la Ley 14/2011, de 1 de julio, de la Ciencia, la Tecnología y la Innovación (LCTI), que distingue entre ciencia y tecnología, por un lado, e innovación, por otro, para cumplir con los dictados constitucionales y también para distinguir entre ambas: la innovación no se limita al conocimiento, sino que lo desborda (57). Tradicionalmente, la conexión entre ciencia, tecnología e innovación ha sido lineal, es decir, una cadena compuesta por tres eslabones, con una dinámica diferenciada y con una influencia en el siguiente a modo de flujo de materiales (58); actualmente, esta concepción se ha superado y se pretende diseñar un sistema de ciencia y tecnología basado en el conocimiento y un sistema de innovación que no se circunscriba al mismo, sino que incluya también un conjunto de procesos asociados a la educación, al mercado y a la competitividad, entre otros (59). De todos modos, tras la recomendación del ERAC Peer Review of the Spanish Research and Innovation system 2014(60), las Estrategias de ID y de Innovación se han unificado para mejorar la coordinación entre ambas. Ciertamente, ello favorece la consonancia entre ambos campos, aunque en la práctica también diluye la financiación que se aporta a cada uno de ellos, puesto que por separado quizás ésta fuera superior. A todo este reparto de competencias entre Estado y comunidades debe añadirse un nuevo actor, que interviene de manera decisiva en el fomento de la investigación: las universidades (artículo 27.10 CE). Según ha concretado el TC, tanto el Estado como las CCAA deben respetar un espacio que les permita adoptar decisiones sobre la investigación que desarrollan (STC 26/1987) (61).

(57) Así el explica MUÑOZ, E., en «La crisis de la política científica: patologías degenerativas y terapias regenerativas. A modo de epílogo». ARBOR Ciencia, Pensamiento y Cultura, julio-agosto 2009, p. 846.

(58) Vale la pena añadir que el plazo innovación se utiliza para describiendo Fenómenos muy diferentes, desde los Descubrimientos Científicos ta el denominación "thinking outside the box", pasando por la creatividad o el diseño. La OCDE Describe cuatro tipos de innovación: innovación tecnológica (productos y procesos) y no tecnológica (marketing y Organización); sobre el concepto de innovación en la OCDE Reducir texto <br> verse Regiones and innovation policy ..., cit., pp. 1-2.

(59) Sobre los motivos que justifican la disociación ver DíEZ BUESO, L., "La gobernanza del sistema español de ciencia, tecnología e innovación", Revista Bioética y Derecho, mayo 2013, pp. 20-27. Así mismo, un amplio análisis sobre los Espacios de la investigación y la innovación puede verse en FERNÁNDEZ, M., Y OTROS, "La gobernanza de la política científica y tecnológica en España». Documentos de trabajo Red CTI-CSIC, septiembre 2008, pp. 5-8.

(60) ERAC Peer Review of the Spanish Research and Innovation system 2014. Comisión Europea, Bruselas-Madrid, agosto 2014.

(61) CUETO PÉREZ, M.: «Incidencia de la nueva Ley de la Ciencia en el régimen jurídico de las Universidades", RAP, N . 187, enero-abril 2012, págs. 269-304 
A partir de los dictados constitucionales, los Estatutos de autonomía concretan el ámbito de actuación de las CCAA y especifican en algunos casos la forma de articulación de las relaciones de colaboración con el Estado (62). Precisamente en el terreno de la colaboración en política científica, ésta sigue los parámetros habituales que se replican en otros campos, respondiendo al bilateralismo como filosofía y a la suscripción de convenios verticales como instrumento específico de relación. Pese al avance que supuso la Ley estatal 13/1986, de 14 de abril, de fomento y coordinación general de la investigación científica y técnica, es unánime la consideración de que no cuidó ni potenció la creación de un sistema científico coordinado entre Estado y comunidades. El principal órgano que debía asegurar tal coordinación era el Consejo General Ciencia y Tecnología, pero este se diseñó fundamentalmente como órgano consultivo de la Comisión estatal Interministerial con unas funciones residuales. Esta configuración, el interés relativo de coordinación del Estado y la dinámica de las propias CCAA provocaron que este Consejo tuviera una influencia escasa dentro del sistema, a excepción de alguna fase de reactivación (63).

La vigente LCTI, en cambio, potenció el Consejo de Política Científica, Tecnológica e Innovación como órgano de coordinación general. Su composición varía leve pero significativamente respecto de su predecesor, pues se compone de titulares de varios ministerios, bajo la presidencia del ministro del ramo, y en el caso de las CCAA, sus representantes no necesariamente deben tener el rango de consejeros. Esta composición posibilita la presencia de los intereses de los distintos ministerios implicados en la materia. También permite a las comunidades decidir libremente a quién designar como representante, lo cual puede venir determinado por su estructura gubernamental o variar en función de los temas, más o menos técnicos, a tratar; además, las CCAA pueden enviar a más de un representante, aunque podrán emitir sólo un voto por comunidad.

Pero, sobre todo, la LCTI avanza en las funciones del Consejo: si bien en textos previos a su aprobación el Consejo sólo «informaba» y «cono-

(62) Un estudio sobre cómo se han asumido las competencias autonómicas en investigación en los distintos Estatutos de autonomía puede encontrarse en GÓMEZ, M., "Administración e investigación científica..., cit., pp. 250-262.

(63) La limitada tarea coordinadora del Consejo se explica en GARCíA, A., y otros, "Legislar sobre política científica para el siglo XXI en España: un nuevo marco normativo para la política de I+D». ARBOR Ciencia, Pensamiento y Cultura, septiembre-octubre 2007, p. 641, al relacionar con el trabajo desarrollado por el Consejo y sobre cierta reactivación en su actividad desde 2006. Véase DíEZ BUESO, L., "¿Hacia una política científica coordinada entre Estado y CCAA?». En SANZ, L., y CRUZ, L.: La investigación y sus actores: institutos y Centros de I + D y sus desafíos. Fundación CyD, 12/2010, p. 212. También en CUETO, M., "La Ley de la ciencia y la coordinación en materia de investigación", madrimasd.org, 2006. 
cía», respectivamente, los dos principales instrumentos estatales de planificación del sistema, la Estrategia de Ciencia y Tecnología y la Estrategia de Innovación, en el texto vigente este órgano "elabora e informa» las propuestas de ambas Estrategias. Es cierto que se trata de una redacción un tanto confusa, debido probablemente al proceso de negociación durante la tramitación de la Ley, pero no cabe duda de que deberá existir una intervención cualitativa de los dos niveles políticos en la aprobación de los documentos que van a guiar los destinos de la política científica de España. Si bien el Estado dispone de la mitad de los votos del Consejo, también es cierto que necesitará el apoyo de las CCAA para alcanzar los dos tercios requeridos para aprobar las Estrategias. Frente a la colaboración orgánica y como sucede en el resto de materias, en política científica prevalecen los convenios verticales bilaterales, que tienen generalmente como finalidad el asesoramiento, la asistencia o la financiación a las CCAA. En este marco, destacan los convenios que crean centros o institutos de investigación por acuerdo entre las universidades, las administraciones autonómicas y la estatal (generalmente sus Organismos Públicos de Investigación, OPIs). Finalmente, el Estado también puso en marcha ciertos programas en los que participan las CCAA, como el vigente Programa de Apoyo a la Innovación de las PYMEs Inno Empresa (2007-2013), diseñado por el gobierno español, financiado por Estado, CCAA y Fondo Europeo de Desarrollo Regional (FEDER) y gestionado por las comunidades.

\section{V.2. La gobernanza de la ciencia}

En España el órgano donde se sitúa la dirección de la política científica ha variado habitualmente en los últimos años, debido a reestructuraciones gubernamentales o a cambios de color en el partido de gobierno (64). Tras las elecciones generales de 2012 esta materia se ubicó en el Ministerio de Economía y competitividad, como Secretaría de Estado de Investigación, Desarrollo e Innovación. Por debajo de esta Secretaría se sitúa la Secretaría general de Ciencia, Tecnología e Innovación, de la que dependen dos Direcciones Generales, la de Investigación Científica y Técnica y la de Innovación y Competitividad. Completa los órganos directivos la Comisión Delegada de Política Científica y Técnica, prevista en la LCTI y que

(64) Sobre las estructuras gubernamentales previas a la actual puede consultarse DíEZ BUESO, L., GÓMEZ, M., y CUETO, M., "La regulación de la universidad en el Estado autonómico». Informe CYD 2008. La contribución de las universidades españolas al desarrollo. Fundación Conocimiento y Desarrollo, 2009, pp. 301-302; y DÍEZ BUESO, L., GÓMEZ, M., y CUETO, M., "La regulación de la universidad en el Estado autonómico». Informe CYD 2009. La contribución de las universidades españolas al desarrollo. Fundación Conocimiento y Desarrollo, 2010, p. 353. 
tiene como funciones principales elevar al Gobierno el Plan Nacional de Investigación, Desarrollo e Innovación Tecnológica, para su aprobación y posterior remisión a las Cortes Generales; ser informada sobre el seguimiento anual del Plan, así como aprobar sus modificaciones; y coordinar las actividades de investigación que los distintos ministerios y los organismos de titularidad estatal realicen en ejecución del Plan Nacional.

Desde el punto de vista del asesoramiento, el Consejo Asesor de Ciencia, Tecnología e Innovación es el órgano de participación de la comunidad científica y tecnológica y de los agentes económicos y sociales, con una regulación que pretende alejarlo de la influencia que previamente tuvo del gobierno central: el número de sus miembros y su elección corre ahora a cargo del Consejo de Política Científica, Tecnológica y de Innovación, que también elegirá a su presidente; además, el reglamento del Consejo Asesor se aprueba a propuesta de sus miembros para asegurar su calidad, independencia y transparencia. En la misma línea, la LCTI intensifica y concreta las funciones de este Consejo respecto de las que tenía anteriormente, aumentando así su peso específico: el espectro de las mismas es doble, por un lado, intervenir en el diseño de las políticas públicas $y$, por otro, configurarse como correa de transmisión de la sociedad en materia científica.

A nivel de gestión, la Fundación Española para la Ciencia y Tecnología (FECYT) es una fundación pública adscrita al Ministerio y se configura como un agente transversal del sistema de la ciencia que trabaja especialmente en su divulgación y en la compra consorciada de información científica; en este último sentido, destaca dentro de su estructura el Observatorio ICONO, que recoge y analiza los principales indicadores y estrategias de $\mathrm{I}+\mathrm{D}+\mathrm{i}$ a nivel autonómico, estatal e internacional. También a nivel de gestión y dependiendo directamente de la Secretaría General como entidad pública empresarial existe el Centro para el Desarrollo Tecnológico Industrial (CDTI), que promueve la innovación y el desarrollo tecnológico de las empresas españolas canalizando sus solicitudes de proyectos de I+D+i en los ámbitos estatal e internacional.

La mayor parte de los organismos de implementación de la política científica dependen directamente de la Secretaría de Estado. Destaca por encima del resto el Consejo Superior de Investigaciones Científicas (CSIC), recientemente configurado como agencia. Se trata de la mayor institución pública dedicada a la investigación en España abarcando desde la investigación básica a la transferencia al sector productivo, además de gestionar un conjunto de importantes infraestructuras. El motor de su investigación lo conforman sus centros e institutos, distribuidos por todas las CCAA, y sus más de 15.000 trabajadores. También dependen de la Secretaría de Estado los ya citados OPIs que, junto con el CSIC y las universidades, forman el núcleo básico del sistema público español de in- 
vestigación, pues ejecutan la mayor parte de las actividades programadas en el Plan Nacional. Estos OPIs son el Centro de Investigaciones Energéticas, Medioambientales y Tecnológicas (CIEMAT), el Instituto de Astrofísica de Canarias (IAC), el Instituto Nacional de Investigación y Tecnología Agraria y Alimentaria (INIA), el Instituto Español de Oceanografía (IEO) y el Instituto Geológico y Minero de España (IGME). Aunque, sin duda, uno de los OPI más destacados es el Instituto de Salud Carlos III (ISCIII), que gestiona, financia y ejecuta la investigación biomédica, a través de tres líneas: el fomento y desarrollo de este sector de investigación; la prestación de servicios científico-técnicos a la administración estatal y al Sistema Nacional de Salud; y la formación científico-técnica sanitaria a través de programas orientados, fundamentalmente, a los profesionales de la salud. Junto a todos estos organismos de implementación de la ciencia a nivel estatal, existe una miríada de centros, de carácter público y privado, vinculados o no a la universidad o a los ministerios, sectoriales o multidisciplinares, con o sin ánimo de lucro, que llevan a cabo I+D+i (65).

Finalmente y como se acaba de avanzar, en España las universidades son uno de los principales protagonistas en la implementación de la $\mathrm{I}+\mathrm{D}+\mathrm{i}(66)$ y en su regulación y funcionamiento intervienen tres instancias (67). El Estado fija a través de su legislación básica los conocimientos indispensables para la obtención de títulos; el régimen básico y la coordinación de los planes de estudio; las bases sobre becas y ayudas; los requisitos básicos para crear universidades; y la regulación de las estructuras básicas de las mismas. Las CCAA desarrollan las bases sobre creación y reconocimiento de universidades y coordinan las de su competencia, incluyendo la readscripción de centros; desarrollan las bases en materia de becas y ayudas; y fijan el calendario académico. Las universidades disponen de capacidades instrumentales derivadas de la autonomía universitaria, que se traducen en las siguientes facultades: dotarse de normas de régimen interno, muy señaladamente los Estatutos; desarrollar la legislación básica relativa a las estructuras y a los órganos universitarios; autoorganizar los medios de que disponga; aprobar los planes de estudio;

(65) Una definición y mapa completo de todos los organismos que llevan a cabo actividades científicas en España puede encontrarse en el Mapa de Instituciones I+D+i de la página web de la FECYT (www.fecyt.es/mapalDI).

(66) Un estudio que relaciona el sistema universitario y el de investigación en España puede verse en DÍEZ BUESO, L., "El sistema universitario y de investigación en España: una perspectiva territorial». Istituzioni del Federalismo, núm. 2, 2012, pp. 423-442. También en CUETO PÉREZ, M.: "La universidad y la investigación» en Comentario a la Ley Orgánica de Universidades, J.V. GONZÁLEZ (dir.), Thomson-Civitas, Madrid, 2009, págs. 695 y ss.

(67) Sobre esta distribución de competencias puede leerse J.C. NAVARRO, Universidades: sistemas europeo, estatal y autonómico. Su articulación competencial. Valencia, Tirant lo Blanch, 2005. 
establecer títulos y diplomas propios; y proveer de vacantes del profesorado mediante concurso, incluyendo la valoración de méritos para contratarlo. Teniendo en cuenta la distribución competencial descrita, para conocer la estructura y la gobernanza de las universidades en España debemos analizar, principalmente, la Ley Orgánica 6/2011, de 21 de diciembre, de Universidades (LOU), y los Estatutos de cada universidad: la primera ofrece cierta homogeneidad al sistema y los segundos concretan las disposiciones de la Ley Orgánica, dando como resultado unas estructuras específicas en cada una de las distintas universidades(68). La LOU establece que toda universidad debe disponer preceptivamente de ciertos órganos, a los que caracteriza mínimamente para que sean los Estatutos los que acaben de completar su regulación; las universidades podrán añadir a estos órganos otros facultativos, cuando sean considerados necesarios para el desarrollo de sus funciones. Así, toda universidad estará integrada por Facultades, Departamentos e Institutos Universitarios de Investigación, a la cabeza de los cuales se encuentran los decanos y los directores de Departamento o Instituto, y cuyos órganos de gobierno son la Junta de Facultad y los Consejos de Departamento.

Como se aprecia, son numerosas las instancias que intervienen en la gobernanza universitaria, de manera que para conseguir una adecuada coordinación la LOU prevé dos órganos de carácter nacional: la Conferencia General de Política Universitaria, con presencia del Estado y de las Comunidades Autónomas y el Consejo de Universidades, con presencia del Estado y de las diferentes universidades.

\section{V.3. La planificación de la política científica}

En materia de planificación la LCTI distingue entre dos Estrategias, la de Ciencia y Tecnología y la de Innovación, y lo hace por dos motivos, el competencial y la distinta aproximación que requieren ambos sectores (69). Estos motivos justifican las escasas diferencias de regulación entre estas Estrategias. La primera diferencia es la previsión de instrumen-

(68) Sobre la estructura de la universidad española puede verse CUESTA, J., "La estructura de las universidades» en Comentario a la Ley Orgánica de Universidades, cit. pp. 251-278. Sobre la gobernanza puede leerse PAREJO, L., "El sistema de gobierno universitario» igualmente en Comentario a la Ley orgánica de Universidades, cit., pp. 205-250.

(69) Sobre los dos motivos que justifican la disociación puede leerse DíEZ BUESO, L., "La gobernanza del sistema español de ciencia, tecnología e innovación». Revista Bioética y Derecho, mayo 2013, pp. 20-27. Vale la pena puntualizar que el término innovación se utiliza para describir fenómenos muy diferentes, desde los descubrimientos científicos hasta el denominado "thinking outside the box", pasando por la creatividad o el diseño. La OCDE describe cuatro tipos de innovación: innovación tecnológica (productos y procesos) y no tecnológica (marketing y organización); sobre el concepto de innovación en la OCDE puede verse Regions and innovation policy..., cit., pp. 1-2. 
tos de coordinación estatal en la Estrategia de Ciencia y Tecnología, inexistentes en la de Innovación; como ya se ha avanzado, ello es consecuencia del sistema de reparto competencial establecido en la $C E$, que atribuye la coordinación general al Estado en el primer terreno pero no en el segundo. La otra diferencia responde al distinto ámbito en que se mueve cada Estrategia: el de la innovación no se limita al conocimiento sino que lo desborda, por lo que en la definición de esta Estrategia se incluyen, también, ejes prioritarios relativos a "la modernización del entorno financiero, el desarrollo de mercados innovadores, las personas, la internacionalización de las actividades innovadoras y la cooperación territorial» (artículo 7.1.b LCTI). Al margen de estas diferencias, ambas Estrategias deberán recoger un marco de referencia plurianual para alcanzar un conjunto de objetivos generales y de prioridades, al tiempo que han de establecer los mecanismos y criterios de articulación de todos los agentes del sistema. Todo ello servirá para orientar el contenido de los Planes estatales y autonómicos que posteriormente se aprueben. De este modo, se pretende superar el modelo basado en la demanda espontanea de la derogada Ley de la ciencia de 1986, al poner el acento en la orientación política identificando los ejes básicos de actuación a largo plazo.

La Estrategia Española de Ciencia, Tecnología e Innovación 2013-2020 ha sido la primera aprobada al amparo de la vigente LCTI por el gobierno estatal, que como se ve ha optado por conjugar en un solo documento lo que en principio deberían haber sido dos Estrategias distintas. Su aprobación se produjo tras un proceso de elaboración donde participaron todos los agentes implicados en el sistema científico, además de las CCAA: tal como disponen los artículos 6.2 y 7.2 LCTI, "el Ministerio de Ciencia e innovación, en colaboración con el Consejo de Política Científica, Tecnológica y de Innovación, elaborará la Estrategia Española de Ciencia y Tecnología, la someterá a informe del propio Consejo, del Consejo Asesor, de los órganos de planificación económica de la administración general del Estado, de la Comisión Delegada del Gobierno para Política Científica, Tecnológica y de Innovación y de los órganos que resulten procedentes, y elevará al Gobierno para su aprobación y posterior remisión a las Cortes Generales». En la Estrategia se recogen los objetivos a seguir y las reformas a acometer en distintos sectores "desde la idea de mercado", que se alinean con los perseguidos por la UE en la Innovation Union y en Horizonte 2020 (70). Estos mismos objetivos deberán servir de referencia para

(70) La dimensión europea resulta fundamental para el desarrollo científico español y no ha sido todavía totalmente explotada, por lo que es especialmente importante esta articulación entre la Estrategia y las políticas de la UE. Sobre la descentralización de la política científica a nivel europeo y su imbricación con las políticas nacionales y autonómicas puede verse CASAS, L., "La descentralización de la política científica». En: SEBASTIÁN, J., RAMOS, I., y FERNÁNDEZ, M., (Eds.), ¿Hacia dónde va la política científica (y tecnológica) en España?. CSIC, 2008, pp. 104-129. 
elaborar los Planes de ciencia y tecnología, estatal y autonómicos, que concretarán los instrumentos y los recursos que deberán utilizarse para alcanzarlos.

La Estrategia se estructura en cinco principios básicos, cuatro objetivos generales, seis ejes prioritarios y otros seis mecanismos de articulación. Los principios son la coordinación de las distintas políticas científicas, incluidas las de la UE; la definición de un marco estable de planificación; la aplicación de criterios de calidad y de impacto social; la eficiencia y rendición de cuentas; y la incorporación de la perspectiva de género. Los objetivos generales se reconducen al reconocimiento y promoción del talento y su empleabilidad; la excelencia; el impulso del liderazgo empresarial en I+D+i; y la orientación del I+D+i a los retos sociales. Los ejes prioritarios tienen un carácter transversal y son la definición de un entorno favorable al I+D+i; la especialización y la agregación de capacidades; la transferencia y gestión del conocimiento en entornos abiertos y flexibles de colaboración; la internacionalización y promoción del liderazgo internacional; la especialización inteligente de los territorios, entendido como el desarrollo regional a partir de sus capacidades(71); y la difusión de la cultura científica a la sociedad. Finalmente, los mecanismos de articulación son la corresponsabilidad de todas las administraciones públicas implicadas en la consecución de los objetivos; el acceso abierto a publicaciones y resultados; la puesta en marcha de un sistema integrado de información; la simplificación administrativa; la armonización de criterios y prácticas de evaluación; y las reformas dirigidas a la captación de financiación privada. Desde el punto de vista de las prioridades temáticas, la Estrategia incluye acciones especiales relacionadas con tecnologías estratégicas como la biotecnología y la nanotecnología y también establece los retos sociales como prioridad temática. Estos retos se relacionan, por ejemplo, con la necesidad de asegurar reformas estructurales para transformar el perfil industrial del país, de un modelo de producción primaria a un sistema de producción basado en la innovación; también se considera un reto dar un giro en los intereses de la investigación pública, pasando de una investigación marcada por la voluntad del científico a otra determinada por la demanda.

En el marco de esta Estrategia, el Gobierno aprobó el Plan estatal de investigación científica, técnica y de innovación 2013-2016, optando de nuevo por unificar los dos Planes previstos en la LCTI en uno sólo en coherencia con lo sucedido con la Estrategia Española. Este Plan también

(71) Con respecto a las estrategias de especialización inteligente, cabe destacar el caso del País Vasco que, desde la década de los 80 del siglo xx, lleva aplicando esta lógica. Ver ARANGUREN QUEREJETA, M.J., MAGRO MONTERO, E., VALDALISO GAGO, J.M., «Estrategias de especialización inteligente: el caso del País Vascon, Innovación y Competitividad, 869, 2012, pp. 65-80. 
se adoptó tras la participación de todos los agentes implicados y una vez "conocido" por el Consejo de Política Científica, Tecnológica y de Innovación (artículo 8.2 LCTI). La estructura del Plan responde a los objetivos y prioridades de la Estrategia y está integrado por cuatro programas estatales: Programa de promoción e incorporación del talento y empleabilidad; Programa de fomento de la investigación científica y técnica de excelencia; Programa de liderazgo empresarial en I+D+i; y Programa de I+D+i orientada a los retos de la sociedad. Estos programas se despliegan en un total de 18 subprogramas de carácter plurianual, que se desarrollarán principalmente mediante convocatorias en concurrencia competitiva en las que deberán detallarse las modalidades de participación y financiación. Además, el Plan estatal recoge dos Acciones Estratégicas: la Acción Estratégica de Salud y la Acción Estratégica de Sociedad y Economía Digital (72).

Con respecto a la consideración del género en la política científica española, cabe señalar que la LCTI incluye aspectos sobre igualdad de género en los procesos de decisión. La Disposición Adicional Decimotercera establece que todas las instituciones y comités regulados por la ley y los comités de selección del sistema de I+D+i deberán seguir los principios establecidos por la Ley Orgánica 3/2007, de 22 de marzo, para la igualdad efectiva de hombres y mujeres. Lo que implica que ninguno de los dos sexos podrá representar más del $60 \%$ o menos del $40 \%$ del total. Igualmente, se indica que los centros de investigación deberán adoptar en dos años planes para la igualdad de género que tendrán que incluir un seguimiento anual (73). También reconoce la importancia de incluir la dimensión de género en los programas de investigación, incluyendo la definición de prioridades de investigación, los problemas, marcos teóricos, métodos, etc., debiendo promover los estudios con una perspectiva de género y el análisis de la situación de la mujer y promover el reconocimiento de las investigadoras en los grupos de investigación. Siguiendo lo indicado en la ley la estrategia estatal incluye la igualdad de género y la dimensión de género como uno de sus cinco principios básicos. Del mismo modo el plan estatal lo considera como una medida horizontal. Sin embargo, el plan no incluye ningún programa específico al respecto y la lista de indicadores para medir su progreso no incluye ningún indicador para medir la igualdad de género en I+D+i.

(72) El Plan de Actuación Anual 2013 fue publicado el 29 de julio de ese mismo año. En él se despliegan los cuatro programas previstos en el Plan estatal 2013-2016, con la peculiaridad de que las Acciones Estratégicas en Salud y en Sociedad y Economía Digital se incorporan en el Plan orientado a los retos de la sociedad.

(73) A modo de ejemplo, el CSIC aprobó su I Plan de Igualdad entre mujeres y hombres en 2013, en el marco del Plan de Igualdad de la AGE y sus organismos públicos 


\section{V.4. Colaboración científica público-privada y transferencia de conocimiento y resultados}

En España la colaboración científica público-privada y la transferencia de conocimiento y resultados es un asunto sobre el que el gobierno estatal ha centrado su atención, sobre todo recientemente, para que las tasas de transferencia sean acordes con la capacidad de generación de conocimiento del sistema público de investigación(74). Desde el punto de vista normativo, han sido dos los hitos recientes en este campo: la aprobación de la LCTI y de la Ley 2/2011, de 4 de marzo, de Economía Sostenible (LES) (75). La primera Ley incorpora importantes novedades sobre colaboración y transferencia que afectan a las universidades y a sus agentes de ejecución (institutos, parques, fundaciones, etc.); a las modalidades de contratación del personal investigador y a su movilidad; y a los instrumentos de colaboración público-privados. Por su parte, el capítulo dedicado a la ciencia y a la innovación de la LES ha supuesto una profunda reforma en el marco legal de la transferencia, por ejemplo, cuando regula las transmisiones a terceros de los derechos sobre los resultados de la actividad investigadora o la cooperación de los agentes públicos con el sector privado a través de las empresas innovadoras de base tecnológica (76). Junto a estas dos Leyes, la modificación de la LOU operada por la Ley 4/2007, de 12 abril, pretendió beneficiar la colaboración público-privada y la transferencia en el campo de las spin-off universitarias, al prever un régimen que favorece la participación del personal docente universitario en las spin-off creadas a partir de proyectos de investigación desarrollados en la universidad. No obstante, parece que las universida-

(74) Un amplio estudio monográfico sobre la situación actual de la colaboración público-privada y la transferencia en España puede encontrarse en TESTAR, X., "La transferencia de tecnología y conocimiento universidad-empresa en España: Estado actual, retos y oportunidades». Monográfico: La transferencia de tecnología y conocimiento universidad-empresa en España. Documentos CYD 17/2012, Fundación Conocimiento y Desarrollo, 2012, pp. 4-8; y también en MANJARRÉS, L., y CARRIÓN, A., "Relaciones universidad-empresa y producción científica de los académicos». En: SANZ, L., y CRUZ, L. (comp.), Análisis sobre la ciencia e innovación en España. Instituto de Políticas y Bienes Públicos (IPP) del CSIC, 2010.

(75) Pese a reconocer la loable intención de ambas Leyes, también se han manifestado críticas por parte de los agentes de la ciencia sobre la incertidumbre que en la actualidad genera el vigente marco normativo; en este sentido, puede leerse RODRIGO, I., «Anexo. La nueva OTRI. Un impulso necesario para un modelo de éxito». Monográfico: La transferencia de tecnología y conocimiento universidad-empresa en España. Documentos CYD 17/2012, Fundación Conocimiento y Desarrollo, 2012, p. 68.

(76) Sobre la incidencia de la LES en la colaboración público-privada y en la transferencia puede verse CASTILLO, J. y HAARICH, N., "Papel de los parques científicos y tecnológicos en la transferencia de conocimiento". Monográfico: La transferencia de tecnología y conocimiento universidad-empresa en España. Documentos CYD 17/2012, Fundación Conocimiento y DesarroIlo, 2012. p. 64. 
des españolas no están orientando sus esfuerzos hacia este medio de transferencia (77).

Desde el punto de vista orgánico, la colaboración y la transferencia se Ilevan a cabo en los Parques Científicos y Tecnológicos(78), en las Plataformas Tecnológicas (79) y en los Centros Tecnológicos o Centros de Apoyo a la Innovación Tecnológica. Aunque en España la transferencia la realizan tradicionalmente las Oficinas de Transferencia de Resultados de la Investigación (OTRIs). Estas Oficinas fueron concebidas en el I Plan Nacional de I+D 1988-1991 como instrumento catalizador de las relaciones entre universidad y empresa, a modo de unidades de transferencia de conocimiento de las universidades y OPIs (80). Concretamente, la OTRI es el interlocutor con empresas y otros agentes socioeconómicos que identifica a los expertos más adecuados para atender sus necesidades; promociona el catálogo de resultados de investigación disponibles para ser transferidos a la sociedad; apoya la firma de contratos entre la universidad/OPI y la empresa; ayuda a encontrar fuentes de financiación pública para las actividades de colaboración entre grupos de la universidad/OPI y otras entidades; gestiona las patentes y otras formas de protección de la I+D+i; y asiste en las actividades encaminadas a la creación de empresas basadas en la explotación del conocimiento generado en la universidad/ OPI(81).

Desde el punto de vista de los programas, el gobierno español ha puesto en marcha actuaciones diversas para apoyar la colaboración y la transferencia. El vigente Plan Estatal 2013-2016 establece una «Línea Ins-

(77) Más detalles sobre la situación previa a la reforma y sobre el contenido concreto de este régimen puede encontrarse en Informe CYD 2009, pp. 197-198; también en esta referencia se reflexiona sobre la falta de uso de este instrumento por las universidades españolas. Datos actualizados sobre las spin-off en España pueden encontrarse anualmente en el Informe CyD. La contribución de las universidades españolas al desarrollo, Fundación Conocimiento y Desarrollo. También en CUETO PÉREZ, M.: «La Universidad y la investigación», cit., pp. 773 y ss.

(78) A nivel estatal existe la Asociación de Parques Científicos y Tecnológicos (APTE), que coordina las empresas e instituciones pertenecientes a la red de Parques españoles. Datos actualizados sobre la actividad de la APTE pueden encontrarse anualmente en el Informe CyD. La contribución de las universidades españolas al desarrollo, Fundación Conocimiento y Desarrollo.

(79) Estas Plataformas se promueven por las empresas, pero se financian en parte por el ministerio del ramo; sobre su configuración y ventajas puede verse Informe CYD 2009. La contribución de las universidades españolas al desarrollo. Fundación Conocimiento y Desarrollo, 2010, pp. 195-196.

(80) En 1996 se crea el Registro de OTRIs, que amplía el concepto al abrir la inscripción a otras entidades del sistema nacional de innovación, como centros tecnológicos, asociaciones empresariales y distintos tipos de fundaciones con actividad en gestión de la ID y transferencia.

(81) Un análisis sobre el modelo de las OTRI en España puede encontrarse en RODRIGO, I., "Anexo. La nueva OTRI..., cit., pp. 67-68. Cabe mencionar también la existencia de la Red OTRI, incardinada en la Conferencia de Rectores de Universidades Españolas, que tiene como objetivo dinamizar las relaciones entre los entornos científicos y productivos; datos actualizados sobre la tarea desarrollada por la Red OTRI pueden encontrarse anualmente en el Informe CyD. La contribución de las universidades españolas al desarrollo, Fundación Conocimiento y Desarrollo. 
trumental de Utilización del Conocimiento y Transferencia Tecnológica» (LIA), que reagrupa todos los instrumentos cuyos objetivos estratégicos sean los siguientes: la transferencia de tecnología desde los organismos de investigación a las empresas o entre estas últimas; la valorización del conocimiento producido en los organismos de investigación; y el fomento a la creación de empresas basadas en el conocimiento. Según se detalla, el gobierno tiene previsto potenciar o poner en marcha una serie de instrumentos: el Estatuto de la Joven Empresa Innovadora, dirigido a empresas jóvenes de tamaño pequeño con una intensidad investigadora elevada, para que dispongan de una financiación limitada en el tiempo y proporcional al gasto en ID certificado en años anteriores; proyectos de creación y consolidación de nuevas empresas tecnológicas, con el propósito de garantizar la inversión inicial de PYMEs tecnológicas y apoyar las actividades de creación y desarrollo de empresas innovadoras de base tecnológica ubicadas en los parques científicos y tecnológicos; mecanismos de apoyo a estos parques, que podrán actuar como Entidades Colaboradoras de las Administraciones Públicas; y, finalmente, instrumentos de apoyo a proyectos para la solución de problemas tecnológicos detectados por empresas, especialmente PYMES.

\section{Conclusiones}

Los factores que condicionan el papel de Estado y regiones en política científica son diversos. Un elemento decisivo es el reparto competencial en esta materia (concurrente en Alemania y de fomento en Austria y España), pero también ciertas competencias estatales relacionadas con la ciencia o la economía que dotan de mayor margen a la autoridad central, así como la configuración y la dinámica del sistema general de descentralización política (de tareas conjuntas en Alemania o de preeminencia del Estado en Austria). Dentro de este marco, resulta fundamental la voluntad de los gobiernos regionales en el momento de desplegar sus capacidades (que difiere considerablemente en un mismo país), al igual que resulta clave la voluntad estatal en el funcionamiento del sistema de colaboración (en tanto en Alemania ha sido clara tanto a nivel de programas como de financiación y gestión del sistema científico, en Austria y España el Estado trata a las regiones como un agente más del sistema(82); en el caso de Reino Unido la coordinación se articula eficazmente a través de los RC).

(82) GONZÁLEZ habla de la conveniencia de concertar una política global entre Estado y regiones para construir una política científica potente y así no favorecer las líneas de investigación de otros países de la Unión en GONZÁLEZ, A., "Organización territorial del Estado en Ciencia y Tecnología. Propuestas para la integración de políticas públicas». Revista Madrid I + D, Extra 1, Monográfico: 20 años de la Ley de Ciencia, 2006. 
En cualquier caso, el despliegue de las políticas científicas en los países estudiados ha conducido al crecimiento de sus estructuras de gobernanza, provocando la aparición de entramados complejos. Esta complejidad aboca a la conveniencia de reformular estas estructuras (83), a partir de evaluaciones previas de resultados y sin olvidar que la complejidad es intrínseca a la gobernanza de la ciencia, pues intervienen gran número de agentes de naturaleza muy diversa que deben disponer de un espacio propio. En la estructuración de la gobernanza resulta determinante la decisión de situar la dirección de la política científica en un departamento u otro (se vinculan a la ciencia/educación en Reino Unido y a la economía en España, en tanto que a ambos en Alemania), pues de este departamento dependerán las asignaciones presupuestarias así como la colaboración intergubernamental y con el resto de actores del sistema; además, la ubicación de la política científica reforzará o debilitará el triángulo del conocimiento (educación, investigación e innovación (84)) y deberá evitar la excesiva diversificación (como recientemente sucedía en Austria). Finalmente, la gobernanza de la política científica debe cumplir otro requisito vital para su éxito, que no es otro que su estabilidad, que conviene extender a los mecanismos de financiación (como sucede en Alemania y Reino Unido); esta estabilidad se ha combinado con un margen de flexibilidad y autonomía de los agentes del sistema (85), que ha derivado en la creación de agencias (con un peso muy relevante en Reino Unido y Alemania en el ámbito de la gestión, así como en España en el de la implementación de la ciencia).

Como acaba de evidenciarse, son muchos los actores del sistema de investigación e innovación que deben integrarse en las estructuras de gobernanza científica. Esta integración también debe producirse a través de los instrumentos de planificación de la política científica, como advirtió la UE al diseñar un proceso concreto de elaboración de las RIS3 que asegura la participación de todos los agentes. Además de estas estrategias, en materia de planificación han resultado básicos otros instrumentos: algunos complementan directamente las estrategias científicas (UK Industrial Strategy en Reino Unido); otros son planes sectoriales (en tecnologías punta o economía ecológica en Alemania, dedicados a la igualdad de

(83) Existe acuerdo en que se debe mejorar la gobernanza de los sistemas científicos de los países de la UE; en este sentido puede leerse Hervás, F. y Muleros, F., Connecting dotes. How to strength the EU knowledge economy. ERAWATCH, 2009, pp. 10 y 12-13.

(84) Sobre la importancia del triángulo del conocimiento en el sistema de la ciencia y su vinculación con la gobernanza científica puede verse Hervás, F. y muleros, F., Connecting dotes ..., pp. 1-7.

(85) En relación con la necesaria adaptabilidad del sistema y la conveniencia de ofrecer autonomía y flexibilidad a sus agentes puede consultarse ACHESON, H., IZSAC, K., MARKIANIDOU, P. y TSIPOURI, L., Innovation policy trends in the EU and beyond. Pro Inno Europe, 2011, pp. 20-21. 
género en Austria); y otros consisten en roadmaps de infraestructuras científicas (Alemania). Resultan también interesantes los instrumentos que implementan de forma parcial las estrategias (Proyectos orientados de futuro alemanes) y cabe reseñar que a veces el complemento a la planificación se concreta en unas determinadas estructuras de gobernanza (las Catapultas en Reino Unido).

Respecto al contenido de las estrategias, cabe señalar que excepcionalmente han fijado unas prioridades claras (Alemania) sino que las orientaciones temáticas son bastante genéricas (España, Austria y Reino Unido), aunque se observa cierta tendencia hacia la priorización de los retos sociales (envejecimiento, calidad de vida, salud) y la ecología (cambio climático, escasez de recursos, energía) (86). La tendencia a la priorización se ha visto reforzada por las RIS3, ya que la financiación europea depende directamente de que la planificación estatal y regional se ajuste a las orientaciones marcadas por la UE; pese a ello todavía queda camino por recorrer, pues las RIS3 aprobadas hasta el momento establecen unos ámbitos de especialización en función de las características de la región, pero junto a ellos recogen también otros muchos campos, probablemente para no condenar a los sectores minoritarios a la ausencia de financiación europea.

La escasa priorización temática de las estrategias y, en ocasiones, su falta de realismo complican la comprobación del cumplimiento de sus objetivos, a lo que se añade la dificultad para obtener datos contrastados y sistematizados; ello ha obstaculizado la puesta en marcha del Open Method of Co-ordination (OMC) en el ámbito de la ciencia (87). En todo caso, este tipo de evaluaciones resulta del todo conveniente y los órganos que las realizan habitualmente las completan con la propuesta de adaptaciones de la estrategia a una realidad siempre cambiante (BMBF Foresight en Alemania).

Las actuaciones en materia de colaboración público-privada y transferencia de conocimiento y resultados son de índole muy variada, tanto por su número como por el tipo de instrumentos utilizados. Entre ellos se encuentran programas específicos para los diferentes estadios de la transferencia (los VTO en Austria o los Innovation Vouchers del Reino Unido); para los diversos actores implicados (EXIST en Alemania) y los nuevos agentes colaboradores (Catapultas del Reino Unido o el Leading-Edge

(86) Sobre la tendencia a concentrar las prioridades en los retos sociales y en la ecología se puede ver ACHESON, H. y otros, "Innovation policy ...», cit., pp. 4-8 y 11; en este artículo también se destaca que hay otros temas que cada vez están recibiendo más atención, como el apoyo a las industrias creativas y de diseño.

(87) A este déficit relativo a los datos y a la dificultad de aplicar la OMC en el terreno de la ciencia se refiere Kaiser, R. y Prange, H., «Managing diversity ...», cit., pp. 249-253 y 261. 
Cluster Competition alemán); y para diferentes ámbitos de actuación, como el sector privado (créditos a bajo interés, compra pública innovadora, capital riesgo e incentivos fiscales en todos los países). Esta variedad de instrumentos se ha puesto en marcha en muy distinto grado y destacan positivamente los acercamientos más "estratégicos", donde la colaboración y la transferencia son objeto de tratamiento específico en documentos de planificación (TSB en Reino Unido).

Los aspectos que han incidido en el éxito de estos instrumentos de fomento han sido dos. Por un lado, la eficacia de los mecanismos de transferencia viene muy determinada por la estructura industrial y la tradición de las colaboraciones público-privadas previas (destaca claramente Alemania). Por otro lado, la evaluación de los instrumentos implementados ha resultado clave para poder corregir las actuaciones de fomento y mejorar así su eficiencia (destacan Alemania y Reino Unido, muy positivamente en este último caso dada la función de aprendizaje de la evaluación por encima de la de control (88)).

Trabajo recibido el 28 de octubre de 2015

Aceptado por el Consejo de Redacción el 22 de enero de 2016

(88) Así se explica ampliamente a MOLAS-GALLART, J., «Research Governance and the Role of Evaluation: A Comparative Study», American Journal of Evaluation, (2012), pp. 1-16. 


\section{Abreviaturas}

Austrian Academy of Sciences y la Ludwig Boltzman Society (LBG)

Austrian Association of Technology Centers (VTO)

Austrian Business Service (AWS)

Austrian Cooperative Research Institutes (ACR-Institutes)

Austrian Institute of Economic Research (WIFO)

Austrian Institute of Technology (AIT)

Austrian Research Promotion Agency (FFG),

Austrian Science Fund (FWF)

Centro de Investigaciones Energéticas, Medioambientales y Tecnológicas

(CIEMAT)

Centro para el Desarrollo Tecnológico Industrial (CDTI)

Christian Doppler Research Association Austria (CDG).

Comunidades autónomas (CCAA)

Consejo Superior de Investigaciones Científicas (CSIC)

Constitución Española (CE)

Department for Business Innovation and Skills (BIS)

Department for Employment and Learning (DELNI)

European Research Area (ERA)

Expert Commission on Research and Innovation (EFI)

Fondo Europeo de Desarrollo Regional (FEDER)

Fraunhofer Society (FHG)

Fundación Española para la Ciencia y Tecnología (FECYT)

Further Education Funding Council (FEFCO)

German Science Council (WR)

High-Tech Strategy 2020 for Germany (HTS 2020)

Industrial Collective Research for SMEs (IGF)

Innovation Union Scoreboard (IUS)

Institute for Advanced Studies Austria (IHS).

Instituto de Astrofísica de Canarias (IAC)

Instituto de Salud Carlos III (ISCIII)

Instituto Español de Oceanografía (IEO)

Instituto Geológico y Minero de España (IGME).

Instituto Nacional de Investigación y Tecnología Agraria y Alimentaria (INIA)

Institutos no Universitarios de Investigación (PROSA)

Investigación, el desarrollo y la innovación (I+D+i)

IPR (Intellectual Property Rights),

Higher Education Funding Council for England (HEFCE)

Higher Education Funding Council for Wales (HEFCW)

Higher Education Innovation Fund (Heifer)

Innovation and Research Strategy for Growth 2011 (IRS) 
Institute of Science and Technology (IST Austria),

Leibniz Association (WGL)

Joint Strategy Group (JSG)

Ley 2/2011, de 4 de marzo, de Economía Sostenible (LES)

Ley 14/2011, de 1 de junio, de la Ciencia, la Tecnología y la Innovación (LCTI)

Ley Orgánica 6/2011, de 21 de diciembre, de Universidades (LOU)

Línea Instrumental de Utilización del Conocimiento y Transferencia Tecnológica (LIA)

Max Planck Society (MPG)

Ministerio de Ciencia e Investigación Austria (BMWF)

Ministerio de Ciencia, Investigación y Economía Austria (BMWFW),

Ministerio de Economía, Familia y Juventud Austria (BMWFJ)

Ministerio de Economía y Tecnología Alemania (BMWi)

Ministerio de Educación e investigación Alemania (BMBF)

Ministerio de Transporte, Innovación y Tecnología Austria (BMVIT).

Oficinas de Transferencia de Resultados de la Investigación (OTRIs)

Open Method of Co-ordination (OMC)

Organismos públicos de investigación (OPIS)

Organización para la Cooperación y el Desarrollo Económico (OCDE)

Pequeñas y Medianas Empresas (PYMEs)

Research Councils (RC)

Research Councils UK (RCUK)

Research Innovation Strategies for Smart Specialisation-RIS3

Science and Tecnology Act 1965 (STA)

Scottish Funding Council (SFC)

SentenciaTribunal Constitucional (STC)

Technology Strategy Board (TSB)

Tribunal Constitucional (TC)

Unión Europea (UE) 
LABURPENA: $\mathrm{I}+\mathrm{G}+\mathrm{b}$ sistemak Europar Batasuneko beste herrialde batzuetan (Alemanian, Austrian eta Erresuma Batuan) zer-nola egituratzen diren konparatu eta aztertzea da lan honen helburua, Espainiako eredua testuinguru horretan kokatuz. Horretarako, honako hauek aztertuko dira, alor horretan eraginkorrak izateko oinarrizko elementu bezala: arlo horren inguruko eskumenen banaketa Estatuaren eta bestelako erakundeen artean, gobernamendua eta politika publikoen plangintza, lankidetza publiko-pribatuko formulak, eta ezagutza eskualdatzea.

GAKO HITZAK: I+G+b. Politika publikoak. Gobernamendua.

RESUMEN: Este trabajo tiene como objetivo llevar a cabo un estudio comparado sobre la articulación de los sistemas de I+D+i en otros países de la Unión Europea como son Alemania, Austria y Reino Unido, situando el modelo español en este contexto. Par ello se atenderá a la distribución de competencias en esta materia entre el Estado y el resto entidades, a la gobernanza y a la planificación de las políticas públicas, así como a las fórmulas de colaboración público-privadas y a la transferencia de conocimiento como elementos fundamentales para alcanzar la eficiencia en este ámbito de actuación.

PALABRAS CLAVES: I+D+i. Políticas públicas. Gobernanza.

ABSTRACT: This work seeks to carry out a comparative study on the organisation of $R+D+i$ systems in other European Union countries such as Germany, Austria and United Kingdom, placing the Spanish model in that context. To do his, it will be taken into consideration the allocation of powers in this field among State and the rest of entities, the governance and the planning of public policies together with the public-private formulas of collaboration and the knowledge transfer as crucial elements for achieving efficiency within this policy field.

KEYWORDS: R+D+i. Public policies. Governance. 